\title{
Non-linear sensorless indirect adaptive speed control of induction motor with unknown rotor resistance and load
}

\author{
Yu-Chao $\mathrm{Lin}^{1}$, Li-Chen $\mathrm{Fu}^{1,2, *}$ and Chin-Yu Tsai ${ }^{1}$ \\ ${ }^{1}$ Department of Electrical Engineering, National Taiwan University, Taipei, Taiwan, R.O.C. \\ ${ }^{2}$ Department of Computer Science and Information Engineering, National Taiwan University, Taipei, Taiwan, R.O.C.
}

\begin{abstract}
SUMMARY
In this paper, a nonlinear indirect adaptive sensorless speed and flux tracking controller for induction motors is proposed. In the controller, only the stator currents are assumed to be measurable. Flux observers and rotor speed estimator are designed to relax the need to flux and speed measurement. Besides, two estimators are also designed to overcome drifting problem of the rotor resistance and unknown load torque. Rigorous stability analysis based on Lyapunov theory is also performed to guarantee that the controller designed here is stable. Computer simulations and experiments are done to demonstrate the performance of our design. Copyright (C) 2000 John Wiley \& Sons, Ltd.

KEY WORDS: indirect adaptive control; induction motor; sensorless
\end{abstract}

\section{INTRODUCTION}

\subsection{Motivation and related researches}

The induction motor is a coupled system with highly non-linear dynamics. In its five states, only stator currents are measurable in sensorless control problem making it difficult to be solved by the engineers. Besides, drift of system parameter drifting is also another problem to cope with. Due to the highly non-linear dynamics, the control of induction motors becomes a popular problem for investigating the relevant non-linear theory and adaptive control over the past decade. In the early years, all system states are assumed to be measurable and parameters are assumed to be known. Under these assumptions, techniques such as classical field orientation [1] and input-output linearization $[2,3]$ are utilized to design the controller. Especially, the controller in Reference [3] was adaptive with respect to both the load and the rotor resistances. Since in these schemes the flux sensors are required, this makes it impractical for implementation. Therefore, flux observers are then designed to relax the need of flux measurement $[4,5]$. These flux observers are designed under the assumption that the rotor resistance is known. Generally, the value of the rotor resistance may drift due to the heating of the rotor, and observers proposed

* Correspondence to: Li-Chen Fu, Department of Electrical Engineering, National Taiwan University, Taipei, Taiwan, R.O.C.

Copyright (C) 2000 John Wiley \& Sons, Ltd. 
above are sensitive to such resistance value. Therefore, efforts have been made to design estimators of the rotor resistance and/or other system parameters [6, 7]. Following these researches, further efforts were then to design controllers and flux observers which are adaptive with respect to both system parameters and/or the load [8-11].

All the schemes above require speed measurement. However, the speed measuring device is rather costly relative to the price of an induction motor in general. Besides, the measured signals are usually noisy and difficult to deal with. Therefore, controllers that do not require speed measurement are obviously preferable for practical implementation. The problem which designs a speed controller of induction motors without rotational transducers then becomes widely interesting and is usually referred to as sensorless control problem. The first paper regarding sensorless control problem can be traced back to 1981. In that paper, the vector control technique is utilized. After that many research results on sensorless vector control have been proposed $[12,13]$, in which analyses are mainly based on the steady-state behaviour and only rough proofs are supplied. On the other hand, in References $[14,15]$, many researches on sensorless control were discussed and compared, including vector control and other modern control theory, such as robust and model reference adaptive system (MRAS). Here, we follow this trend to design a fully non-linear adaptive sensorless speed controller for induction motors based on flux observer.

\subsection{Organization of this paper}

In this paper, an introduction of induction motors and related researches are discussed in Section 1. In Section 2, the mathematical model of an induction motor will be presented. The main part of this paper is Section 3 in which observers and controller are designed and proved in detail. The computer simulations and experimental results are presented in Section 4. Finally, we will make some conclusions in Section 5.

\section{PRELIMINARIES AND PROBLEM FORMULATION}

In this chapter, we introduce the induction motor model and our control problem.

\subsection{Motor model}

If the induction motor never goes into the saturation region, and the air-gap MMF is sinusoidal, then it can be characterized by the following dynamic equations [16]:

$$
\begin{aligned}
L_{\mathrm{o}} \dot{I}_{a} & =-M R_{\mathrm{r}} I_{a}-\beta_{1} I_{a}+R_{\mathrm{r}} \psi_{a}+\beta_{2} \omega_{\mathrm{r}} \psi_{b}+\beta_{3} V_{a} \\
L_{\mathrm{o}} \dot{I}_{b} & =-M R_{\mathrm{r}} I_{b}-\beta_{1} I_{b}-\beta_{2} \omega_{\mathrm{r}} \psi_{a}+R_{\mathrm{r}} \psi_{b}+\beta_{3} V_{b} \\
L_{\mathrm{r}} \dot{\psi}_{a} & =-R_{\mathrm{r}} \psi_{a}+M R_{\mathrm{r}} I_{a}-\beta_{2} \omega_{\mathrm{r}} \psi_{b} \\
L_{\mathrm{r}} \dot{\psi}_{b} & =-R_{\mathrm{r}} \psi_{b}+M R_{\mathrm{r}} I_{b}+\beta_{2} \omega_{\mathrm{r}} \psi_{a} \\
T_{\mathrm{e}} & =k_{\mathrm{T}}\left(\psi_{a} I_{b}-\psi_{b} I_{a}\right)
\end{aligned}
$$

where $L_{\mathrm{o}}, \beta_{1}, \beta_{2}, \beta_{3}$ are constants defined in the nomenclature. The mathematical model listed above is referred to the well-known stator fixed reference frame. The five equations derived above 
can fully describe the dynamics of the electrical part of the motor-load system. Meanwhile, we can derive the dynamics of the mechanical part through some analysis based on Newton's law

$$
J \dot{\omega}_{\mathrm{r}}+D \omega_{\mathrm{r}}+T_{\mathrm{L}}=T_{\mathrm{e}}
$$

where $J>0$ is the rotor inertia, $D>0$ is the damping coefficient and $T_{\mathrm{L}}$ is the load torque. After successfully deriving the dynamics of the whole system, we begin to introduce our control problems and objects.

\subsection{Problem formulation}

In this paper, we try to design a speed controller for induction motors, which does not rely on any shaft sensor outputs and only measures the stator currents. All the parameters of the motor except the rotor resistance and the load torque are assumed to be known. The only knowledge about the rotor resistance we have is its nominal value. Given this information about the motor, for any speed and flux commands which are second-order continuously differentiable bounded functions, this controller should be able to drive the motor to track the commands.

\section{INDUCTION MOTOR CONTROL}

Before a thorough investigation on the observers and controllers, several assumptions will be presented below to make the problem more precise.

\section{Assumption}

(A1) All parameters of the motor are known, except the rotor resistance $R_{\mathrm{r}}$.

(A2) The stator currents are measurable.

(A3) The load torque $T_{\mathrm{L}}$ is an unknown constant.

(A4) The desired rotor speed should be a bounded smooth function with known first and second-order time derivatives.

Assumption (A1) comes from the uncertainty of the rotor resistance. Its value may vary up to 100 percent during the operation due to the change in the temperature and/or working condition. Assumption (A2) is a realistic consideration in the actual application of the induction motors. The reason why we only measure the stator currents is stated in the introduction of this thesis. Finally, assumptions (A3) and (A4) are also quite realistic in practical applications.

Control objective: Given the desired rotor speed trajectory $\omega_{\mathrm{rd}}(t)$, our control goal is to design control laws such that the rotor speed $\omega_{\mathrm{r}}$ and the rotor flux $\Psi$ can track their respective desired trajectories asymptotically in time with all internal signals being bounded, subject to assumptions (A1)-(A4).

\subsection{Observer design}

In order to measure the rotor fluxes, sensors have to be inserted into the air gap. This requires re-designing or re-assembling of the motor. Obviously, this contradicts to the advantages of the 
induction motors, i.e. low cost and easy fabrication. Therefore, here we design observers to observe the rotor flux and relax the need to flux sensors. Besides, as mentioned in the introduction of this paper, the rotor speed is not measured, either. This leads us to construct a speed observer as well. In addition to the rotor flux observers and the rotor speed observer, we build observers to observe the stator currents despite they being are readily measurable signals. These additional current observers provide us extra information useful for designing our non-linear adaptive subsequently. We then have five observers in total in our system to reconstruct the five system states. Moreover, since variation of the rotor resistance may cause undesirable deterioration of performance, a rotor resistance estimator is built as well. These observers and estimators will be discussed in the following contents.

The system model is shown in Section 2. We set $R_{\mathrm{r}}=R_{\mathrm{rn}}+\theta_{1}, \omega_{\mathrm{r}}=\omega_{\mathrm{rd}}+\theta_{2}$ where $\theta_{1}$ stands for (unknown) difference between the actual resistance value and its nominal value, and $\theta_{2}$ denotes the speed tracking error. For easy reference, we define the notations for the observed values and the observation errors as $\tilde{I}_{a}=I_{a}-\hat{I}_{a}, \tilde{I}_{b}=I_{b}-\hat{I}_{b}, \tilde{\psi}_{a}=\psi_{a}-\hat{\psi}_{a}, \tilde{\psi}_{b}=\psi_{b}-\hat{\psi}_{b}$, $\tilde{R}_{\mathrm{r}}=R_{\mathrm{r}}-\hat{R}_{\mathrm{r}}, \tilde{\omega}_{\mathrm{r}}=\omega_{\mathrm{r}}-\hat{\omega}_{\mathrm{r}}$ where the symbol denotes that it is an observed value and the symbol $\sim$ denotes an observation error. According to the structure of the dynamics in (1), the observers are proposed as in Reference [8]:

$$
\begin{aligned}
& L_{\mathrm{o}} \dot{\hat{I}}_{a}=k_{0} \tilde{I}_{a}-\left(M \hat{R}_{\mathrm{r}}+\beta_{1}\right) I_{a}+\hat{R}_{\mathrm{r}} \hat{\psi}_{\mathrm{a}}+\beta_{2} \hat{\omega}_{\mathrm{r}} \hat{\psi}_{b}+\beta_{3} V_{a}+v_{1}+v_{5} \\
& L_{\mathrm{o}} \hat{\hat{I}}_{b}=k_{0} \tilde{I}_{b}-\left(M \hat{R}_{\mathrm{r}}+\beta_{1}\right) I_{b}-\beta_{2} \hat{\omega}_{\mathrm{r}} \hat{\psi}_{a}+\hat{R}_{\mathrm{r}} \hat{\psi}_{b}+\beta_{3} V_{b}+v_{2}+v_{6} \\
& L_{\mathrm{r}} \hat{\psi}_{a}=-\hat{R}_{\mathrm{r}} \hat{\psi}_{a}+M \hat{R}_{\mathrm{r}} I_{a}-\beta_{2} \hat{\omega}_{\mathrm{r}} \hat{\psi}_{b}+v_{3} \\
& L_{\mathrm{r}} \dot{\hat{\psi}}_{b}=-\hat{R}_{\mathrm{r}} \hat{\psi}_{b}+M \hat{R}_{\mathrm{r}} I_{b}+\beta_{2} \hat{\omega}_{\mathrm{r}} \hat{\psi}_{a}+v_{4}
\end{aligned}
$$

where $\hat{R}_{\mathrm{r}}=R_{\mathrm{r} n}+\hat{\theta}_{1}, \hat{\omega}_{\mathrm{r}}=\omega_{\mathrm{rd}}+\hat{\theta}_{2}$ and the constant $k_{0}>0$ is a control gain. Finally, the speed observer and the rotor resistance estimator, which are not shown here, will be presented toward the end of this section based on Lyapunov stability analysis.

\subsection{Analysis of the observer}

With the observers designed above, in this section we will proceed to analyse their stability. First, we derive the observation error dynamics by subtracting (3) from (1) to yield

$$
\begin{aligned}
& L_{\mathrm{o}} \dot{\tilde{I}}_{a}=-k_{0} \tilde{I}_{a}+R_{\mathrm{r}} \tilde{\psi}_{a}+\tilde{R}_{\mathrm{r}} \hat{\psi}_{a}-M \tilde{R}_{\mathrm{r}} I_{a}+\beta_{2} \omega_{\mathrm{r}} \tilde{\psi}_{b}+\beta_{2} \tilde{\omega}_{\mathrm{r}} \tilde{\psi}_{b}-v_{1}-v_{5} \\
& L_{\mathrm{o}} \dot{\tilde{I}}_{b}=-k_{0} \tilde{I}_{b}+R_{\mathrm{r}} \tilde{\psi}_{b}+\tilde{R}_{\mathrm{r}} \hat{\psi}_{b}-M \tilde{R}_{\mathrm{r}} I_{b}-\beta_{2} \omega_{\mathrm{r}} \tilde{\psi}_{a}-\beta_{2} \tilde{\omega}_{\mathrm{r}} \tilde{\psi}_{a}-v_{2}-v_{6} \\
& L_{\mathrm{r}} \dot{\tilde{\psi}}_{a}=-R_{\mathrm{r}} \tilde{\psi}_{a}-\tilde{R}_{\mathrm{r}} \hat{\psi}_{a}-\beta_{2} \omega_{\mathrm{r}} \tilde{\psi}_{b}-\beta_{2} \tilde{\omega}_{\mathrm{r}} \hat{\psi}_{b}+M \tilde{R}_{\mathrm{r}} I_{a}-v_{3} \\
& L_{\mathrm{r}} \tilde{\psi}_{b}=-R_{\mathrm{r}} \tilde{\psi}_{b}-\tilde{R}_{\mathrm{r}} \hat{\psi}_{b}+\beta_{2} \omega_{\mathrm{r}} \hat{\psi}_{a}+\beta_{2} \tilde{\omega}_{\mathrm{r}} \hat{\psi}_{a}+M \tilde{R}_{\mathrm{r}} I_{b}-v_{4}
\end{aligned}
$$

After a careful review of the above equations, an interesting property of these observation errors can be found, i.e. there are a lot of common terms in the error dynamics of the stator currents and in those of the rotor fluxes, but they are with opposite signs. In fact, this phenomenon also appears in the system model (1). In order to utilize this property to cancel the unmeasurable 
terms, we design two auxiliary observation errors [8]:

$$
\begin{aligned}
& \tilde{Z}_{a}=L_{\mathrm{o}} \tilde{I}_{a}+L_{\mathrm{r}} \tilde{\psi}_{a} \\
& \tilde{Z}_{b}=L_{\mathrm{o}} \tilde{I}_{b}+L_{\mathrm{r}} \tilde{\psi}_{b}
\end{aligned}
$$

Another interesting characteristic is studied here. Although the auxiliary observation errors, $\tilde{Z}_{a}$ and $\tilde{Z}_{b}$, are not measurable (because they are composed of $\tilde{I}$ and $\tilde{\psi}$, and $\tilde{\psi}$ is not measurable), their first-order time derivatives are measurable. This special characteristic comes from the cancellation of the common terms mentioned above.

Two additional error signals $\eta_{a}$ and $\eta_{b}$ are then defined as follows:

$$
\begin{gathered}
\eta_{a}=\tilde{Z}_{a}-\zeta_{a} \\
\eta_{b}=\tilde{Z}_{b}-\zeta_{b}
\end{gathered}
$$

where $\zeta_{a}$ and $\zeta_{b}$ are auxiliary control signals. Again, $\eta_{a}$ and $\eta_{b}$ are unmeasurable errors but with measurable first-order time derivatives. It will be clear later that the use of these two signals will bring help to cope with the coupling terms of the unmeasurable signals, such as $\omega_{\mathrm{r}} \psi$.

Motivated by how the coupling terms can be cancelled, we design the observer inputs $v_{1}$ and $v_{2}$ to be

$$
\begin{aligned}
& v_{1}=-\frac{\hat{R}_{\mathrm{r}}}{L_{\mathrm{r}}}\left(L_{\mathrm{o}} \tilde{I}_{a}-\zeta_{a}\right)-\frac{\beta_{2} \hat{\omega}_{\mathrm{r}}}{L_{\mathrm{r}}}\left(L_{\mathrm{o}} \tilde{I}_{b}-\zeta_{b}\right) \\
& v_{2}=-\frac{\hat{R}_{\mathrm{r}}}{L_{\mathrm{r}}}\left(L_{\mathrm{o}} \tilde{I}_{b}-\zeta_{b}\right)+\frac{\beta_{2} \hat{\omega}_{\mathrm{r}}}{L_{\mathrm{r}}}\left(L_{\mathrm{o}} \tilde{I}_{a}-\zeta_{a}\right)
\end{aligned}
$$

by (5) and (6), which can be further rearranged into

$$
\begin{aligned}
& v_{1}=\frac{\hat{R}_{\mathrm{r}}}{L_{\mathrm{r}}}\left(L_{\mathrm{o}} \tilde{I}_{a}-\zeta_{a}\right)+\frac{\beta_{2} \hat{\omega}_{\mathrm{r}}}{L_{\mathrm{r}}}\left(L_{\mathrm{o}} \tilde{I}_{b}-\zeta_{b}\right)-\frac{R_{\mathrm{r}}}{L_{\mathrm{r}}} \eta_{a}-\frac{\beta_{2} \omega_{\mathrm{r}}}{L_{\mathrm{r}}} \eta_{b}+R_{\mathrm{r}} \tilde{\psi}_{a}+\beta_{2} \omega_{\mathrm{r}} \tilde{\psi}_{b} \\
& v_{2}=\frac{\hat{R}_{\mathrm{r}}}{L_{\mathrm{r}}}\left(L_{\mathrm{o}} \tilde{I}_{\mathrm{b}}-\zeta_{\mathrm{b}}\right)-\frac{\beta_{2} \tilde{\omega}_{\mathrm{r}}}{L_{\mathrm{r}}}\left(L_{\mathrm{o}} \tilde{I}_{a}-\zeta_{a}\right)-\frac{R_{\mathrm{r}}}{L_{\mathrm{r}}} \eta_{b}+\frac{\beta_{2} \omega_{\mathrm{r}}}{L_{\mathrm{r}}} \eta_{a}+R_{\mathrm{r}} \tilde{\psi}_{b}-\beta_{2} \omega_{\mathrm{r}} \tilde{\psi}_{a}
\end{aligned}
$$

Substituting (8) into the first two equations of (4), we get the error dynamics about current observation as

$$
\begin{aligned}
L_{\mathrm{o}} \dot{\tilde{I}}_{a}= & -k_{0} \tilde{I}_{a}+\frac{\hat{R}_{\mathrm{r}}}{L_{\mathrm{r}}} \eta_{a}+\frac{\beta_{2} \hat{\omega}_{\mathrm{r}}}{L_{\mathrm{r}}} \eta_{b} \\
& +\tilde{R}_{\mathrm{r}}\left(\hat{\psi}_{a}-M I_{a}-\frac{L_{\mathrm{o}}}{L_{\mathrm{r}}} \tilde{I}_{a}+\frac{1}{L_{\mathrm{r}}} \zeta_{a}+\frac{1}{L_{\mathrm{r}}} \eta_{a}\right) \\
& +\tilde{\omega}_{\mathrm{r}}\left(\beta_{2} \hat{\psi}_{b}-\frac{\beta_{2} L_{\mathrm{o}}}{L_{\mathrm{r}}} \tilde{I}_{b}+\frac{\beta_{2}}{L_{\mathrm{r}}} \zeta_{b}+\frac{\beta_{2}}{L_{\mathrm{r}}} \eta_{b}\right)-v_{5}
\end{aligned}
$$




$$
\begin{aligned}
L_{\mathrm{o}} \dot{\tilde{I}}_{b}= & -k_{0} \tilde{I}_{b}+\frac{\hat{R}_{\mathrm{r}}}{L_{\mathrm{r}}} \eta_{b}-\frac{\beta_{2} \hat{\omega}_{\mathrm{r}}}{L_{\mathrm{r}}} \eta_{a} \\
& +\tilde{R}_{\mathrm{r}}\left(\hat{\psi}_{b}-M I_{b}-\frac{L_{\mathrm{o}}}{L_{\mathrm{r}}} \tilde{I}_{b}+\frac{1}{L_{\mathrm{r}}} \zeta_{b}+\frac{1}{L_{\mathrm{r}}} \eta_{b}\right) \\
& +\tilde{\omega}_{\mathrm{r}}\left(-\beta_{2} \hat{\psi}_{a}+\frac{\beta_{2} L_{\mathrm{o}}}{L_{\mathrm{r}}} \tilde{I}_{a}-\frac{\beta_{2}}{L_{\mathrm{r}}} \zeta_{a}-\frac{\beta_{2}}{L_{\mathrm{r}}} \eta_{a}\right)-v_{6}
\end{aligned}
$$

and we define

$$
\begin{aligned}
& \Omega_{11}=\hat{\psi}_{a}-M I_{a}-\frac{L_{\mathrm{o}}}{L_{\mathrm{r}}} \tilde{I}_{a}+\frac{1}{L_{\mathrm{r}}} \zeta_{a}+\frac{1}{L_{\mathrm{r}}} \eta_{a} \\
& \Omega_{12}=\hat{\psi}_{b}-M I_{b}-\frac{L_{\mathrm{o}}}{L_{\mathrm{r}}} \tilde{I}_{b}+\frac{1}{L_{\mathrm{r}}} \zeta_{b}+\frac{1}{L_{\mathrm{r}}} \eta_{b} \\
& \Omega_{21}=\beta_{2} \hat{\psi}_{b}-\frac{\beta_{2} L_{\mathrm{o}}}{L_{\mathrm{r}}} \tilde{I}_{b}+\frac{\beta_{2}}{L_{\mathrm{r}}} \zeta_{b}+\frac{\beta_{2}}{L_{\mathrm{r}}} \eta_{b} \\
& \Omega_{22}=-\beta_{2} \hat{\psi}_{a}+\frac{\beta_{2} L_{\mathrm{o}}}{L_{\mathrm{r}}} \tilde{I}_{a}-\frac{\beta_{2}}{L_{\mathrm{r}}} \zeta_{a}-\frac{\beta_{2}}{L_{\mathrm{r}}} \eta_{a}
\end{aligned}
$$

After investigation on the error dynamics of the stator current observer, we now turn to study of the error dynamics of the rotor flux observer. It is noteworthy that the rotor flux error dynamics will not be analysed directly, but instead the analysis on $\tilde{Z}_{a}$ and $\tilde{Z}_{b}$ will be made.

Let $v_{3}$ and $v_{4}$ be designed as

$$
\begin{aligned}
& v_{3}=-k_{0} \tilde{I}_{a}+\frac{L_{\mathrm{o}}}{L_{\mathrm{r}}}\left(\hat{R}_{\mathrm{r}} \tilde{I}_{a}+\beta_{2} \hat{\omega}_{\mathrm{r}} \tilde{I}_{b}\right) \\
& v_{4}=-k_{0} \tilde{I}_{b}+\frac{L_{\mathrm{o}}}{L_{\mathrm{r}}}\left(\hat{R}_{\mathrm{r}} \tilde{I}_{b}-\beta_{2} \hat{\omega}_{\mathrm{r}} \tilde{I}_{a}\right)
\end{aligned}
$$

So far, we have derived the error dynamics of the observed stator current and the auxiliary errors. Now, we are ready to perform the Lyapunov stability analysis to examine the stability condition of these observers. First, we define a Lyapunov function candidate as

$$
V_{0}=\frac{1}{2} \tilde{I}_{a}^{2}+\frac{1}{2} \tilde{I}_{b}^{2}+\frac{1}{2} k_{n} \eta_{a}^{2}+\frac{1}{2} k_{\eta} \eta_{b}^{2}+\frac{1}{2} k_{\omega} \tilde{\omega}_{\mathrm{r}}^{2}+\frac{1}{2} k_{R} \tilde{R}_{r}^{2}
$$

where control gains $k_{\eta}, k_{\omega}$ and $k_{R}$ should satisfy

$$
k_{\eta}, k_{\omega}, k_{R}>0
$$


Taking the time derivative of $V_{0}$ along the system trajectories, we obtain

$$
\begin{aligned}
\dot{V}_{0}= & -k_{0} \tilde{I}_{a}^{2}-k_{0} \tilde{I}_{b}^{2}-\tilde{I}_{a} v_{5}-\tilde{I}_{b} v_{6}+\frac{\tilde{R}_{\mathrm{r}}}{L_{\mathrm{o}} L_{\mathrm{r}}}\left(\tilde{I}_{a} \tilde{\eta}_{a}+\tilde{I}_{b} \tilde{\eta}_{b}\right)+\frac{\beta_{2} \tilde{\omega}_{\mathrm{r}}}{L_{\mathrm{o}} L_{\mathrm{r}}}\left(\tilde{I}_{b} \tilde{\eta}_{a}+\tilde{I}_{a} \tilde{\eta}_{b}\right) \\
& +\eta_{a}\left[\frac{\hat{R}_{\mathrm{r}}}{L_{\mathrm{r}} L_{\mathrm{o}}} \tilde{I}_{a}-\frac{\beta_{2} \hat{\omega}_{\mathrm{r}}}{L_{\mathrm{r}} L_{\mathrm{o}}} I_{b}+k_{n} \dot{\eta}_{a}\right]+\eta_{b}\left[\frac{\hat{R}_{\mathrm{r}}}{L_{\mathrm{r}} L_{\mathrm{o}}} \tilde{I}_{b}+\frac{\beta_{2} \hat{\omega}_{\mathrm{r}}}{L_{\mathrm{r}} L_{\mathrm{o}}} \tilde{I}_{a}+k_{n} \dot{\eta}_{b}\right] \\
& +\frac{\tilde{R}_{\mathrm{r}}}{L_{\mathrm{o}}}\left[\hat{\Omega}_{11} \tilde{I}_{a}+\hat{\Omega}_{12} \hat{I}_{b}+k_{R} L_{\mathrm{o}} \tilde{\tilde{R}}_{\mathrm{r}}\right]+\frac{\tilde{\omega}_{\mathrm{r}}}{L_{\mathrm{o}}}\left[\hat{\Omega}_{21} \tilde{I}_{a}+\hat{\Omega}_{22} \hat{I}_{b}+k_{\omega} L_{\mathrm{o}} \dot{\tilde{\omega}}_{\mathrm{r}}\right]
\end{aligned}
$$

via definitions of four additional functions $\hat{\Omega}_{11}, \hat{\Omega}_{12}, \hat{\Omega}_{21}$ and $\hat{\Omega}_{22}$ :

$$
\begin{aligned}
& \hat{\Omega}_{11}=\hat{\psi}_{a}-M I_{a}-\frac{L_{\mathrm{o}}}{L_{\mathrm{r}}} \tilde{I}_{a}+\frac{1}{L_{\mathrm{r}}} \zeta_{a}+\frac{1}{L_{\mathrm{r}}} \hat{\eta}_{a} \\
& \hat{\Omega}_{12}=\hat{\psi}_{b}-M I_{b}-\frac{L_{\mathrm{o}}}{L_{\mathrm{r}}} \tilde{I}_{b}+\frac{1}{L_{\mathrm{r}}} \zeta_{b}+\frac{1}{L_{\mathrm{r}}} \hat{\eta}_{b} \\
& \hat{\Omega}_{21}=\beta_{2} \hat{\psi}_{b}-\frac{\beta_{2} L_{\mathrm{o}}}{L_{\mathrm{r}}} \tilde{I}_{b}+\frac{\beta_{2}}{L_{\mathrm{r}}} \zeta_{b}+\frac{\beta_{2}}{L_{\mathrm{r}}} \hat{\eta}_{b} \\
& \hat{\Omega}_{22}=-\beta_{2} \hat{\psi}_{a}+\frac{\beta_{2} L_{\mathrm{o}}}{L_{\mathrm{r}}} \tilde{I}_{a}-\frac{\beta_{2}}{L_{\mathrm{r}}} \zeta_{a}-\frac{\beta_{2}}{L_{\mathrm{r}}} \hat{\eta}_{a}
\end{aligned}
$$

Note that $\hat{\Omega}_{11}, \hat{\Omega}_{12}, \hat{\Omega}_{21}$ and $\hat{\Omega}_{22}$ are combination of all measurable errors, observed values and auxiliary control signals, and hence are known functions. Here, to complete the final analysis an additional assumption is required.

(A5) The speed tracking error and the change of the rotor resistance from its nominal value, i.e. $\theta_{2}$ and $\theta_{1}$, varies slowly so that its first-order time derivative can be negligible, i.e. $\dot{\theta}_{1} \approx 0$, $\dot{\theta}_{2} \approx 0$

Again, if $\dot{\eta}_{a}, \dot{\eta}_{b}, \dot{\hat{R}}_{\mathrm{r}}$ and $\dot{\hat{\omega}}_{\mathrm{r}}$ are designed properly, those terms in the last four brackets in (12) can be cancelled out, which then leads to the design of those signal as

$$
\begin{aligned}
& \dot{\eta}_{a}=-\frac{1}{k_{\eta}}\left(\frac{\hat{R}_{\mathrm{r}}}{L_{\mathrm{r}} L_{\mathrm{o}}} \tilde{I}_{a}-\frac{\beta_{2} \hat{\omega}_{\mathrm{r}}}{L_{\mathrm{r}} L_{\mathrm{o}}} \tilde{I}_{b}\right) \\
& \dot{\eta}_{b}=-\frac{1}{k_{\eta}}\left(\frac{\hat{R}_{\mathrm{r}}}{L_{\mathrm{r}} L_{\mathrm{o}}} \tilde{I}_{b}+\frac{\beta_{2} \hat{\omega}_{\mathrm{r}}}{L_{\mathrm{r}} L_{\mathrm{o}}} \tilde{I}_{a}\right) \\
& \dot{\tilde{R}}_{\mathrm{r}}=-\frac{1}{k_{R}}\left(\frac{\hat{\Omega}_{11}}{L_{\mathrm{o}}} \tilde{I}_{a}+\frac{\hat{\Omega}_{12}}{L_{\mathrm{o}}} \tilde{I}_{b}\right) \\
& \dot{\tilde{\omega}}_{\mathrm{r}}=-\frac{1}{k_{\omega}}\left(\frac{\hat{\Omega}_{21}}{L_{\mathrm{o}}} \tilde{I}_{a}+\frac{\hat{\Omega}_{22}}{L_{\mathrm{o}}} \tilde{I}_{b}\right)
\end{aligned}
$$


where $\hat{\eta}_{a}$ and $\hat{\eta}_{b}$ are estimated values of $\eta_{a}$ and $\eta_{b}$, respectively, satisfying

$$
\begin{array}{ll}
\dot{\hat{\eta}}_{a}=\dot{\eta}_{a}, & \hat{\eta}_{a}(0)=0 \\
\dot{\hat{\eta}}_{b}=\dot{\eta}_{b}, & \hat{\eta}_{b}(0)=0
\end{array}
$$

Evidently, if the estimators $\hat{\eta}_{a}$ and $\hat{\eta}_{b}$ are designed as above, the estimation errors can be easily found to be

$$
\begin{aligned}
& \tilde{\eta}_{a}=\eta_{a}(0) \\
& \tilde{\eta}_{b}=\eta_{b}(0)
\end{aligned}
$$

By substituting the rotor resistance estimator and the rotor speed observer, we can re-assess $\dot{V}$ as

$$
\begin{aligned}
\dot{V}_{\mathrm{o}}= & -k_{0} \tilde{I}_{a}^{2}-k_{0} \tilde{I}_{b}^{2}-\tilde{I}_{a} v_{5}-\tilde{I}_{b} v_{6}+\frac{\tilde{R}_{\mathrm{r}}}{L_{\mathrm{o}} L_{\mathrm{r}}}\left(\tilde{I}_{a} \tilde{\eta}_{a}+\tilde{I}_{b} \tilde{\eta}_{b}\right)+\frac{\beta_{2} \tilde{\omega}_{\mathrm{r}}}{L_{\mathrm{o}} L_{\mathrm{r}}}\left(\tilde{I}_{b} \tilde{\eta}_{a}+\tilde{I}_{b} \tilde{\eta}_{b}\right) \\
\leqslant & -k_{0} \tilde{I}_{a}^{2}-k_{0} \tilde{I}_{b}^{2}-\tilde{I}_{a} v_{5}-\tilde{I}_{b} v_{6}+\frac{1}{L_{\mathrm{r}} L_{\mathrm{o}}}\left|\tilde{I}_{a}\right|\left|\tilde{R}_{\mathrm{r}} \tilde{\eta}_{a}\right|+\frac{\beta_{2}}{L_{\mathrm{r}} L_{\mathrm{o}}}\left|\tilde{I}_{a}\right|\left|\tilde{\omega}_{\mathrm{r}} \tilde{\eta}_{b}\right| \\
& +\frac{1}{L_{\mathrm{r}} L_{\mathrm{o}}}\left|\tilde{I}_{b}\right|\left|\tilde{R}_{\mathrm{r}} \tilde{\eta}_{b}\right|+\frac{\beta_{2}}{L_{\mathrm{r}} L_{\mathrm{o}}}\left|\tilde{I}_{b}\right|\left|\tilde{\omega}_{\mathrm{r}} \tilde{\eta}_{a}\right|
\end{aligned}
$$

Clearly, the upper bound on $\dot{V}$ on the right-hand side (RHS) of Equation (15) still does not have definite negative sign. To cope with that, we incorporate the variable structure design (VSD) into our controller. Let us first assume

$$
\begin{aligned}
& \tilde{\eta}_{a}=\eta_{a}(0) \leqslant \delta_{a} \\
& \tilde{\eta}_{b}=\eta_{b}(0) \leqslant \delta_{b}
\end{aligned}
$$

and derive the four functions $F_{i}(t), i=1,2,3,4$ which satisfy

$$
\begin{aligned}
& F_{1}(t) \geqslant \delta_{a}\left|\tilde{R}_{r}\right|, \quad F_{2}(t) \geqslant \delta_{b}\left|\tilde{\omega}_{\mathrm{r}}\right| \\
& F_{3}(t) \geqslant \delta_{b}\left|\tilde{R}_{r}\right|, \quad F_{4}(t) \geqslant \delta_{a}\left|\tilde{\omega}_{\mathrm{r}}\right|
\end{aligned}
$$

It is not hard to choose the functions $F_{1}(t)$ and $F_{3}(t)$, whereas in Appendix A we show that the bounds $F_{2}(t)$ and $F_{4}(t)$ are calculable. Accordingly, we design the four control signal $v_{5}$ and $v_{6}$ as

$$
\begin{aligned}
& v_{5}=\frac{1}{L_{\mathrm{r}}} \operatorname{sgn}\left(\tilde{I}_{a}\right)\left(F_{1}(t)+\beta_{2} F_{2}(t)\right) \\
& v_{6}=\frac{1}{L_{\mathrm{r}}} \operatorname{sgn}\left(\tilde{I}_{b}\right)\left(F_{3}(t)+\beta_{2} F_{4}(t)\right)
\end{aligned}
$$


where $\operatorname{sgn}(\cdot)$ is the sign function defined as

$$
\operatorname{sgn}(x)=\left\{\begin{array}{ll}
1 & \text { if } x \geqslant 0 \\
0 & \text { if } x<0
\end{array} \quad \forall x \in \mathfrak{R}\right.
$$

From (A5) and (14), we can in turn design an observer for the rotor speed tracking error, an estimator for rotor resistance change, and the auxiliary signals as

$$
\begin{aligned}
& \hat{\theta}_{1}=\frac{1}{k_{R}}\left(\frac{\hat{\Omega}_{11}}{L_{\mathrm{o}}} \tilde{I}_{a}+\frac{\hat{\Omega}_{12}}{L_{\mathrm{o}}} \tilde{I}_{b}\right), \quad \hat{\theta}_{1}(0) \geqslant 0 \\
& \hat{\theta}_{2}=\frac{1}{k_{\omega}}\left(\frac{\hat{\Omega}_{21}}{L_{\mathrm{o}}} \tilde{I}_{a}+\frac{\hat{\Omega}_{22}}{L_{\mathrm{o}}} \tilde{I}_{b}\right) \\
& \dot{\zeta}_{a}=\dot{\tilde{Z}}_{a}-\dot{\eta}_{a} \\
& \dot{\zeta}_{b}=\dot{\tilde{Z}}_{b}-\dot{\eta}_{b}
\end{aligned}
$$

As a result, $\dot{V}_{\text {o }}$ becomes

$$
\dot{V}_{\mathrm{o}} \leqslant-\frac{k_{\mathrm{o}}}{L_{\mathrm{o}}}\left(\tilde{I}_{a}^{2}+\tilde{I}_{b}^{2}\right)
$$

It follows that $\tilde{I}_{a}, \tilde{I}_{b}, \tilde{R}_{\mathrm{r}}, \tilde{\omega}_{\mathrm{r}}, \eta_{a}$ and $\eta_{b}$ are all bounded. We now impose that $\hat{\theta}_{1}(t)+R_{\mathrm{r} n} \geqslant$ $R_{\mathrm{r} n} / 2>0, \forall t \geqslant 0$, by means of incorporating a projection algorithm into the adaptation law given by the first equation in (17). Denoting by $\hat{\theta}_{1 \mathrm{p}}(t)$ the modification of $\hat{\theta}_{1}(t)$ given by the projection algorithm, $\hat{\theta}_{1 \mathrm{p}}(t)$ is chosen such that

$$
\left[\left(R_{\mathrm{r} n}+\theta_{1}\right)-\left(R_{\mathrm{r} n}+\hat{\theta}_{1}(t)\right)\right]^{2} \geqslant\left[\left(R_{\mathrm{r} n}+\theta_{1}\right)-\left(R_{\mathrm{r} n}+\hat{\theta}_{1 \mathrm{p}}(t)\right)\right]^{2}
$$

which guarantees that the value of $V_{\mathrm{o}}$ does not increase when $\hat{\theta}_{1}$ is replaced by $\hat{\theta}_{1 \mathrm{p}}$ given by the projection algorithm. Since $R_{\mathrm{r}}=R_{\mathrm{r} n}+\theta_{1}$ is positive, a simple projection algorithm which guarantees that $(18)$ is satisfied is obtained by reinitializing the estimate $\hat{\theta}_{1}(t)$

$$
\hat{\theta}_{1}(t)=\hat{\theta}_{1 \mathrm{p}}(t)=\frac{-R_{\mathrm{r} n}}{2}+\varepsilon \quad \text { if } \hat{\theta}_{1}(t) \leqslant R_{\mathrm{r} n}
$$

with $\varepsilon>0$ a positive constant such that

$$
\varepsilon \leqslant 2\left(R_{\mathrm{r} n}+\theta_{1}\right)=2 R_{\mathrm{r}}
$$

Let us for the time being assume that $\left(I_{a}, I_{b}\right)$ are bounded in finite time, i.e. $\left(I_{a}, I_{b} \in L_{\infty e}\right)$, then from Appendix A, $\left(\psi_{a}, \psi_{b}, \omega_{\mathrm{r}}\right)$ are also bounded in finite time. Since $\left(\psi_{a}, \psi_{b}, I_{a}, I_{\mathrm{b}}, \omega_{\mathrm{r}}\right)$ are bounded, if $\hat{R}_{\mathrm{r}}=R_{\mathrm{r} n}+\hat{\theta}_{1} \geqslant R_{\mathrm{r} n} / 2>0$, it follows that $\hat{\psi}_{a}$ and $\hat{\psi}_{b}$ are also bounded according to (3), and therefore $\tilde{Z}_{a}$ and $\tilde{Z}_{b}$ are bounded from (5). This implies that $\zeta_{a}$ and $\zeta_{b}$ are bounded and that $v_{1}, v_{2}$, $v_{3}$ and $v_{4}$ are bounded as well. According to (9), $\dot{\tilde{I}}_{a}$ and $\dot{\tilde{I}}_{b}$ are bounded. On the other hand, $\dot{\tilde{I}}_{a}$ and 
$\dot{\tilde{I}}_{b}$ are $L_{2}$ signals, since

$$
\begin{aligned}
\int_{0}^{t}\left(\tilde{I}_{a}^{2}(\tau)+\tilde{I}_{b}^{2}(\tau)\right) \mathrm{d} \tau & =\frac{k_{0}}{L_{0}}(V(t)-V(0)) \\
& \leqslant \frac{k_{0}}{L_{0}} V(0), \quad \forall t \geqslant 0
\end{aligned}
$$

By Barbalat's lemma, it follows that

$$
\lim _{t \rightarrow \infty} \tilde{I}_{a}(t)=0, \quad \lim _{t \rightarrow \infty} \tilde{I}_{b}(t)=0,
$$

We can rewrite (9) and (14) in matrix from as

$$
\begin{gathered}
\dot{I}=A \tilde{I}+W^{\mathrm{T}}(t) X+B(t) \\
\dot{X}=-\Lambda W(t) \tilde{I}+\Lambda C \tilde{I}
\end{gathered}
$$

in which $\tilde{I}=\left[\tilde{I}_{a}, \tilde{I}_{b}\right]^{\mathrm{T}}, X=\left[\tilde{R}_{\mathrm{r}}, \tilde{\omega}_{\mathrm{r}}, \eta_{a}, \eta_{b}\right], \Lambda=\operatorname{diag}\left[1 / k_{\eta}, 1 / k_{\eta}, 1 / k_{R}, 1 / k_{\omega}\right]$ and

$$
\begin{aligned}
A & =\left[\begin{array}{cc}
-\frac{k_{\mathrm{o}}}{L_{\mathrm{o}}} & 0 \\
0 & -\frac{k_{\mathrm{o}}}{L_{\mathrm{o}}}
\end{array}\right] \\
W^{\mathrm{T}}(t) & =\left[\begin{array}{cccc}
\frac{\Omega_{11}}{L_{\mathrm{o}}} & \frac{\Omega_{21}}{L_{\mathrm{o}}} & \frac{R_{\mathrm{r}}}{L_{\mathrm{r}} L_{\mathrm{o}}} & \frac{\beta_{2} \hat{\omega}_{\mathrm{r}}}{L_{\mathrm{r}} L_{\mathrm{o}}} \\
\frac{\Omega_{12}}{L_{\mathrm{o}}} & \frac{\Omega_{22}}{L_{\mathrm{o}}} & -\frac{\beta_{2} \hat{\omega}_{\mathrm{r}}}{L_{\mathrm{r}} L_{\mathrm{o}}} & \frac{\hat{R}_{\mathrm{r}}}{L_{\mathrm{r}} L_{\mathrm{o}}}
\end{array}\right] \\
B(t) & =\left[\begin{array}{cccc}
v_{5} \\
v_{6}
\end{array}\right] \\
C^{\mathrm{T}} & =\left[\begin{array}{cccc}
\frac{\tilde{\eta}_{a}}{L_{\mathrm{r}} L_{\mathrm{o}}} & \frac{\tilde{\eta}_{b}}{L_{\mathrm{r}} L_{\mathrm{o}}} & 0 & 0 \\
\frac{\tilde{\eta}_{b}}{L_{\mathrm{r}} L_{\mathrm{o}}} & \frac{\tilde{\eta}_{a}}{L_{\mathrm{r}} L_{\mathrm{o}}} & 0 & 0
\end{array}\right]
\end{aligned}
$$

To analyse the asymptotic condition of system (20), we introduce the following important working lemma.

\section{Lemma 1.}

If there exist two positive constants $T$ and $\varepsilon$ such that

$$
\int_{t}^{t+T} W(\tau) W^{\mathrm{T}}(\tau) \geqslant \varepsilon I>0, \quad \forall t \geqslant 0
$$


then the equilibrium state $X=0, \tilde{I}=0$ of the following system:

$$
\begin{aligned}
\dot{\tilde{I}} & =A(t) \tilde{I}+W^{\mathrm{T}}(t) X \\
\dot{X} & =-W(t) \tilde{I}
\end{aligned}
$$

are uniformly asymptotically stable.

The proof is shown in Appendix B.

We can get $\tilde{I}$ asymptotically tending to zero from (19), and the matrix $B(t)$ in $(20)$ is bounded. Motivated by Lemma 1 , when $\tilde{I}$ is small, we supply small control signals $\left(v_{5}, v_{6}\right)$ in matrix $B(t)$, but if $\tilde{I}$ tend close to zero, we set $v_{5}, v_{6}$ zero. As a result, we can make Equations (20) and (23) virtually the same. Then, the equilibrium point $(\tilde{I}=0, X=0)$ remains asymptotically stable for such kind of hybrid set-up according to Lemma 1 , namely $\tilde{R}_{\mathrm{r}}, \tilde{\omega}_{\mathrm{r}}, \eta_{a}, \eta_{b}, \tilde{I}_{a}$ and $\widetilde{I}_{b}$ tend asymptotically to zero. By now, let us rewrite the last two terms in Equation (4).

$$
\left[\begin{array}{c}
\hat{\psi}_{a} \\
\hat{\psi}_{b}
\end{array}\right]=\left[\begin{array}{cc}
-R_{\mathrm{r}} / L_{\mathrm{r}} & -\beta_{2} \omega_{\mathrm{r}} / L_{\mathrm{r}} \\
\beta_{2} \omega_{\mathrm{r}} / L_{\mathrm{r}} & -R_{\mathrm{r}} / L_{\mathrm{r}}
\end{array}\right]\left[\begin{array}{c}
\psi_{a} \\
\psi_{b}
\end{array}\right]+\tilde{R}_{\mathrm{r}}\left[\begin{array}{c}
\left(M I_{a}-\hat{\psi}_{a}\right) / L_{\mathrm{r}} \\
\left(M I_{b}-\hat{\psi}_{b}\right) / L_{\mathrm{r}}
\end{array}\right]+\beta_{2} \tilde{\omega}_{\mathrm{r}}\left[\begin{array}{c}
-\hat{\psi}_{b} \\
\hat{\psi}_{a}
\end{array}\right]+\left[\begin{array}{c}
v_{3} \\
v_{4}
\end{array}\right]
$$

where $v_{3}, v_{4}$ are the functions of $\tilde{I}_{a}, \tilde{I}_{b}$ as shown in (11). Since $I_{a}$ and $I_{b}$ are assumed bounded in finite time and the fact that $\hat{\psi}_{a}$ and $\hat{\psi}_{b}$ are bounded (to be clear in the following remark), we can conclude that $\tilde{\psi}_{a}$ and $\tilde{\psi}_{b}$ also tend asymptotically to zero. In conclusions, Equation (3) with $\left(v_{1}, v_{2}\right.$, $\left.v_{3}, v_{4}, v_{5}, v_{6}\right)$ given in (7), (11), and (16) and $\hat{\theta}_{1}, \hat{\theta}_{2}, \eta_{a}$ and $\eta_{b}$ updated according to (17) and (14) constitute a convergent adaptive observer provided that (22) is satisfied.

Remark

According to (3), we can rewrite the dynamics of the observed flux in the form

$$
\left[\begin{array}{c}
\hat{\psi}_{a} \\
\dot{\hat{\psi}}_{b}
\end{array}\right]=\left[\begin{array}{cc}
-\hat{R}_{\mathrm{r}} & -\beta_{2} \hat{\omega}_{\mathrm{r}} \\
\beta_{2} \hat{\omega}_{\mathrm{r}} & -\hat{R}_{\mathrm{r}}
\end{array}\right]\left[\begin{array}{c}
\hat{\psi}_{a} \\
\hat{\psi}_{b}
\end{array}\right]+\left[\begin{array}{cc}
M \hat{R}_{\mathrm{r}} & 0 \\
0 & M \hat{R}_{\mathrm{r}}
\end{array}\right]\left[\begin{array}{l}
I_{a} \\
I_{b}
\end{array}\right]+\left[\begin{array}{l}
v_{3} \\
v_{4}
\end{array}\right]
$$

Because $\left(I_{a}, I_{b}, v_{3}, v_{4}\right)$ are bounded, we only need to check the convergence of the form as follows:

$$
\left[\begin{array}{c}
\dot{\hat{\psi}}_{a} \\
\hat{\psi}_{b}
\end{array}\right]=\left[\begin{array}{cc}
-\hat{R}_{\mathrm{r}} & -\beta_{2} \hat{\omega}_{\mathrm{r}} \\
\beta_{2} \hat{\omega}_{\mathrm{r}} & -\hat{R}_{\mathrm{r}}
\end{array}\right]\left[\begin{array}{c}
\hat{\psi}_{a} \\
\hat{\psi}_{b}
\end{array}\right]
$$

Consider that

$$
V_{\hat{\psi}}=\frac{1}{2}\left(\hat{\psi}_{a}^{2}+\hat{\psi}_{b}^{2}\right)
$$

then

$$
\begin{aligned}
\dot{V}_{\hat{\psi}} & =\hat{\psi}_{a} \dot{\hat{\psi}}_{a}+\hat{\psi}_{b} \dot{\hat{\psi}}_{b} \\
& =-\hat{R}_{\mathrm{r}}\left(\hat{\psi}_{a}^{2}+\dot{\hat{\psi}}_{b}^{2}\right) \leqslant 0
\end{aligned}
$$


which implies the exponential stability condition of the homogeneous system and hence the bounded input bounded state (BIBS) property of the forced (non-homogeneous) system.

\subsection{Controller design}

In the previous section, observers and parameter estimator are designed and their stability properties are analysed based on Lyapunov stability theory. We then use the observed or estimated values in designing a proper controller as if they were true values. In this section, we propose a state feedback control for induction motor system which is adaptive with respect to the unknown constant load torque $T_{\mathrm{L}}$, assuming all states $\left(I_{a}, I_{b}, \psi_{a}, \psi_{b}\right)$ are measurable and the rotor resistance $\left(R_{\mathrm{r}}\right)$ is a known constant. The controller objective is to guarantee asymptotical zero convergence of rotor speed tracking error and rotor flux tracking error. The dynamics model of the induction motor is expressed as (1). And, we define the speed tacking error, flux tacking error and load torque estimation error as $e_{\omega}=\omega_{\mathrm{r}}-\omega_{\mathrm{rd}}, e_{\Psi}=\psi_{a}^{2}+\psi_{d}^{2}-\Psi_{d}^{2}, e_{\mathrm{T}}=T_{\mathrm{L}}-\hat{T}_{\mathrm{L}}$ where $\Psi_{\mathrm{d}}^{2}=\psi_{a \mathrm{~d}}^{2}+\psi_{b \mathrm{~d}}^{2}, \Psi^{2}=\psi_{\mathrm{a}}^{2}+\psi_{\mathrm{b}}^{2}, \omega_{\mathrm{rd}}$ and $\Psi_{\mathrm{d}}$ are the reference signals and $\hat{T}_{\mathrm{L}}$ is a time-varying estimation of $T_{L}$.

With the tracking errors and load torque estimation error defined above, their dynamics can be derived from (1) as

$$
\begin{aligned}
J \dot{e}_{\omega} & =k_{\mathrm{T}}\left(\psi_{a} I_{b}-\psi_{b} I_{a}\right)-D \omega_{\mathrm{r}}-T_{\mathrm{L}}-J \dot{\omega}_{\mathrm{rd}} \\
L_{\mathrm{r}} \dot{e}_{\Psi} & =-2 R_{\mathrm{r}} \Psi^{2}+2 M R_{\mathrm{r}}\left(\psi_{a} I_{a}+\psi_{b} I_{b}\right)-2 L_{\mathrm{r}} \dot{\Psi}_{\mathrm{d}}^{2}
\end{aligned}
$$

By designing the input signal $I_{a}$ and $I_{b}$, we first consider a Lyapunov function candidate defined as

$$
V_{1}=\frac{1}{2}\left(r_{1} J e_{\omega}^{2}+r_{2} L_{\mathrm{r}} e_{\psi}^{2}+r_{3} e_{\mathrm{T}}^{2}\right)
$$

where gains $r_{1}, r_{2}$ and $r_{3}$ are positive.

Taking the time derivative of $V_{1}$, we then get its time derivative according to (24) as

$$
\begin{aligned}
\dot{V}_{1}= & r_{1} e_{\omega}\left(k_{\mathrm{T}}\left(\psi_{a} I_{b}-\psi_{b} I_{a}\right)-D \omega_{\mathrm{r}}-T_{\mathrm{L}}-J \dot{\omega}_{\mathrm{rd}}\right) \\
& +r_{2} e_{\Psi}\left(-2 R_{\mathrm{r}} \Psi^{2}+2 M R_{\mathrm{r}}\left(\psi_{a} I_{a}+\psi_{b} I_{b}\right)-2 L_{\mathrm{r}} \dot{\Psi}_{\mathrm{d}}^{2}\right)+r_{3} e_{\mathrm{T}} \dot{e}_{\mathrm{T}}
\end{aligned}
$$

If we let $I_{a}$ and $I_{b}$ be chosen such that

$$
\begin{aligned}
k_{\mathrm{T}}\left(\psi_{a} I_{b}-\psi_{b} I_{a}\right)-D \omega_{\mathrm{r}}-J \dot{\omega}_{\mathrm{r}}-J \dot{\omega}_{\mathrm{rd}} & =-K_{\omega} e_{\omega}+\hat{T}_{\mathrm{L}} \\
-2 R_{\mathrm{r}} \Psi^{2}+2 M R_{\mathrm{r}}\left(\psi_{a} I_{a}+\psi_{b} I_{b}\right)-2 L_{\mathrm{r}} \dot{\Psi}_{\mathrm{d}}^{2} & =-K_{\Psi} e_{\Psi}
\end{aligned}
$$

with $K_{\omega}>0$ and $K_{\Psi}>0$. From (26), the choice of the currents $I_{a}$ and $I_{b}$ are equivalent to the following:

$$
\begin{aligned}
& I_{a}=\frac{1}{\Psi^{2}}\left(\Omega_{1} \psi_{a}-\Omega_{2} \psi_{b}\right) \\
& I_{b}=\frac{1}{\Psi^{2}}\left(\Omega_{2} \psi_{a}+\Omega_{1} \psi_{b}\right)
\end{aligned}
$$


where

$$
\begin{aligned}
& \Omega_{1}=\frac{2 R_{\mathrm{r}} \Psi^{2}+2 L_{\mathrm{r}} \dot{\Psi}_{\mathrm{d}}^{2}-K_{\Psi} e_{\Psi}}{2 M R_{\mathrm{r}}} \\
& \Omega_{2}=\frac{D \omega_{\mathrm{r} d}+J \dot{\omega}_{\mathrm{rd}}-\left(K_{\omega}-D\right) e_{\omega}+\hat{T}_{\mathrm{L}}}{k_{\mathrm{T}}}
\end{aligned}
$$

But, the currents are not directly available as control inputs. They are related to the actual controls (stator voltages) by the first two equations in (1). To design the actual control inputs $V_{a}$ and $V_{b}$, we thus let currents in (27) to be the desired currents.

$$
\begin{aligned}
& I_{a \mathrm{~d}}=\frac{1}{\Psi^{2}}\left(\Omega_{1} \psi_{a}-\Omega_{2} \psi_{b}\right) \\
& I_{b \mathrm{~d}}=\frac{1}{\Psi^{2}}\left(\Omega_{2} \psi_{a}-\Omega_{1} \psi_{b}\right)
\end{aligned}
$$

Define the current errors, namely, the differences between the actual currents and their desired values, as follows:

$$
\begin{aligned}
& e_{a}=I_{a}-I_{a \mathrm{~d}} \\
& e_{b}=I_{b}-I_{b \mathrm{~d}}
\end{aligned}
$$

Then, the error dynamics involving $\left(e_{\omega}, e_{\Psi}, e_{a}, e_{b}\right)$ can be summerized as follows:

$$
\begin{aligned}
J \dot{e}_{\omega} & =k_{\mathrm{T}}\left(\psi_{a} e_{b}-\psi_{b} e_{a}\right)-K_{\omega} e_{\omega}-e_{\mathrm{T}} \\
L_{\mathrm{r}} \dot{e}_{\Psi} & =2 M R_{\mathrm{r}}\left(\psi_{a} e_{a}+\psi_{b} e_{b}\right)-K_{\Psi} e_{\Psi} \\
L_{0} \dot{e}_{a} & =-M R_{\mathrm{r}} I_{a}-\beta_{1} I_{a}+R_{\mathrm{r}} \psi_{a}+\beta_{2} \omega_{\mathrm{r}} \psi_{b}+\beta_{3} V_{a}-L_{\mathrm{o}} \dot{I}_{a \mathrm{~d}} \\
L_{0} \dot{e}_{b} & =-M R_{\mathrm{r}} I_{b}-\beta_{1} I_{b}-\beta_{2} \omega_{\mathrm{r}} \psi_{\mathrm{a}}+R_{\mathrm{r}} \psi_{b}+\beta_{3} V_{b}-L_{\mathrm{o}} \dot{I}_{b \mathrm{~d}}
\end{aligned}
$$

The derivatives of the reference currents computed from (29) are found as

$$
\begin{aligned}
& \dot{I}_{a \mathrm{~d}}=\Omega_{3}-\frac{L_{0} \psi_{b}\left(K_{\omega}-D\right)}{\Psi^{2} k_{\mathrm{T}} J} e_{\mathrm{T}} \\
& \dot{I}_{b \mathrm{~d}}=\Omega_{4}+\frac{L_{0} \psi_{a}\left(K_{\omega}-D\right)}{\Psi^{2} k_{\mathrm{T}} J} e_{\mathrm{T}}
\end{aligned}
$$


where

$$
\begin{aligned}
& \Omega_{3}=L_{\mathrm{o}}\left(\left(\frac{\mathrm{d}}{\mathrm{d} t} \frac{\psi_{a}}{\Psi^{2}}\right) \Omega_{1}+\frac{\psi_{a}}{\Psi^{2}} \dot{\Omega}_{1}\right)-L_{\mathrm{o}}\left(\left(\frac{\mathrm{d}}{\mathrm{d} t} \frac{\psi_{b}}{\Psi^{2}}\right) \Omega_{2}-\frac{\psi_{b}}{\Psi^{2}} \Omega_{5}\right) \\
& \Omega_{4}=L_{\mathrm{o}}\left(\left(\frac{\mathrm{d}}{\mathrm{d} t} \frac{\psi_{a}}{\Psi^{2}}\right) \Omega_{2}+\frac{\psi_{a}}{\Psi^{2}} \Omega_{5}\right)+L_{\mathrm{o}}\left(\left(\frac{\mathrm{d}}{\mathrm{d} t} \frac{\psi_{b}}{\Psi^{2}}\right) \Omega_{1}+\frac{\psi_{b}}{\Psi^{2}} \dot{\Omega}_{1}\right)
\end{aligned}
$$

in which

$$
\begin{aligned}
\frac{\mathrm{d}}{\mathrm{d} t}\left(\frac{\psi_{a}}{\Psi^{2}}\right) & =\frac{\dot{\psi}_{a} \Psi-2 \psi_{a} \dot{\Psi}}{\Psi^{3}} \\
\frac{\mathrm{d}}{\mathrm{d} t}\left(\frac{\psi_{b}}{\Psi^{2}}\right) & =\frac{\dot{\psi}_{b} \Psi-2 \psi_{b} \dot{\Psi}}{\Psi^{3}} \\
\dot{\Omega}_{1} & =\frac{4 R_{\mathrm{r}} \Psi \dot{\Psi}+2 L_{\mathrm{r}} \dot{\Psi}_{\mathrm{d}} \dot{\Psi}_{\mathrm{d}}+2 L_{\mathrm{r}} \Psi_{\mathrm{d}} \ddot{\Psi}_{\mathrm{d}}-K_{\Psi} \dot{e}_{\Psi}}{2 M R_{\mathrm{r}}} \\
\dot{\Omega}_{2} & =\frac{\left(K_{\omega}-D\right)}{k_{\mathrm{T}} J} e_{\mathrm{T}}-\Omega_{5}
\end{aligned}
$$

with

$$
\Omega_{5}=\frac{-D \dot{\omega}_{\mathrm{rd}}-J \ddot{\omega}_{\mathrm{rd}}+\left[\left(K_{\omega}-D\right) / J\right]\left(k_{\mathrm{T}}\left(\psi_{a} e_{b}-\psi_{b} e_{a}\right)-K_{\omega} e_{\omega}\right)-\hat{T}_{\mathrm{L}}}{k_{\mathrm{T}}}
$$

Only the expression of $\dot{\Omega}_{2}$ depends on the unknown signal $e_{\mathrm{T}}$, but others are all made up of known signal. Finally, we chose the control input terms $V_{a}$ and $V_{b}$ as

$$
\begin{aligned}
& V_{a}=\frac{1}{\beta_{3}}\left(M R_{\mathrm{r}} I_{a}+\beta_{1} I_{a}-R_{\mathrm{r}} \psi_{a}-\beta_{2} \omega_{\mathrm{r}} \psi_{b}+\Omega_{3}-K_{a} e_{a}+u_{a}\right) \\
& V_{b}=\frac{1}{\beta_{3}}\left(M R_{\mathrm{r}} I_{b}+\beta_{1} I_{b}-R_{\mathrm{r}} \psi_{b}+\beta_{2} \omega_{\mathrm{r}} \psi_{a}+\Omega_{4}-K_{b} e_{b}+u_{b}\right)
\end{aligned}
$$

where $K_{a}, K_{b} \geqslant 0$, and $u_{a}$ and $u_{b}$ are to be designed later. Given such design arrangement, the error dynamics of $I_{a}$ and $I_{b}$ become

$$
\begin{aligned}
& L_{\mathrm{o}} \dot{e}_{a}=-K_{a} e_{a}+u_{a}+\frac{L_{\mathrm{o}} \psi_{b}\left(K_{\omega}-D\right)}{\Psi^{2} k_{\mathrm{T}} J} e_{\mathrm{T}} \\
& L_{\mathrm{o}} \dot{e}_{b}=-K_{b} e_{b}+u_{b}-\frac{L_{\mathrm{o}} \psi_{a}\left(K_{\omega}-D\right)}{\Psi^{2} k_{\mathrm{T}} J} e_{\mathrm{T}}
\end{aligned}
$$




\subsection{Analysis of the controller}

Note that the dynamics of the state tracking error $\left(e_{a}, e_{b}, e_{\Psi}, e_{\omega}\right)$ have been explicitly derived in (30) and (35), where the desired signals $\Psi_{\mathrm{d}}$ and $\omega_{\mathrm{rd}}$, are a priori known, and the desired currents are specified in (29). Then, under these circumstance, we can proceed to check the performance of the controller.

The analysis is also based on the Lyapunov stability theory. First, we define a Lyapunov function candidate for the controller as

$$
V=\frac{1}{2}\left(r_{1} J e_{\omega}^{2}+r_{2} L_{\mathrm{r}} e_{\Psi}^{2}+r_{3} e_{\mathrm{T}}^{2}+r_{4} L_{\mathrm{o}}\left(e_{a}^{2}+e_{b}^{2}\right)+\varepsilon e_{\mathrm{T}} e_{\omega}\right)
$$

for some positive constants $r_{1}, r_{2}, r_{3}$ and $r_{4}$ and for some sufficiently small $\in(>0)$ such that the quadratic function

$$
V_{2}\left(e_{\omega}, e_{\mathrm{T}}\right)=\frac{1}{2}\left(r_{1} J e_{\omega}^{2}+r_{3} e_{\mathrm{T}}^{2}+\varepsilon e_{\mathrm{T}} e_{\omega}\right)
$$

is positive definite. So, the Lyapunov candidate function $V$ is indeed positive definite. Using the state tracking error dynamics (30) and (35), we can obtain the time derivative of $V$ as

$$
\begin{aligned}
\dot{V}= & -r_{1} K_{\omega} e_{\omega}^{2}-r_{2} K_{\Psi} e_{\Psi}^{2}-r_{4} K_{a} e_{a}^{2}-r_{4} K_{b} e_{b}^{2} \\
& +e_{\mathrm{T}}\left(r_{1}-e_{\omega}+r_{4} e_{a}\left(K_{\omega}-D\right) \frac{L_{\mathrm{o}} \psi_{b}}{\Psi^{2} k_{\mathrm{T}} J}\right. \\
& \left.-r_{4} e_{b}\left(K_{\omega}-D\right) \frac{L_{\mathrm{o}} \psi_{a}}{\Psi^{2} k_{\mathrm{T}} J}+r_{3} \dot{e}_{\mathrm{T}}+\varepsilon \dot{e}_{\omega}\right) \\
& +e_{a}\left(-r_{1} e_{\omega} k_{\mathrm{T}} \psi_{b}+2 r_{2} e_{\Psi} M R_{\mathrm{r}} \psi_{a}+r_{4} u_{a}\right) \\
& +e_{b}\left(r_{1} e_{\omega} k_{\mathrm{T}} \psi_{b}+2 r_{2} e_{\Psi} M R_{\mathrm{r}} \psi_{b}+r_{4} u_{b}\right)+\varepsilon \dot{e}_{\mathrm{T}} e_{\omega}
\end{aligned}
$$

In order to make $\dot{V} \leqslant 0$, what is left to be decided is $\dot{e}_{\mathrm{T}}$ and $V_{a}, V_{b}$. After a careful choice, we follow the payload estimation algorithm for $\dot{e}_{\mathrm{T}}$ as

$$
\begin{aligned}
\dot{\hat{T}}_{L}= & -\dot{e}_{\mathrm{T}} \\
= & \frac{1}{r_{3}}\left(-r_{1} e_{\omega}+r_{4} e_{a} \frac{L_{\mathrm{o}} \psi_{b}\left(K_{\omega}-D\right)}{\Psi^{2} k_{\mathrm{T}} J}-r_{4} e_{b} \frac{L_{\mathrm{o}} \psi_{a}\left(K_{\omega}-D\right)}{\Psi^{2} k_{\mathrm{T}} J}\right) \\
& +\frac{\varepsilon}{J}\left(k_{\mathrm{T}}\left(\psi_{a} e_{b}-\psi_{b} e_{a}\right)-K_{\omega} e_{\omega}\right)
\end{aligned}
$$


which updates $\dot{V}$ into a slightly different form as

$$
\begin{aligned}
\dot{V}= & -r_{1} K_{\omega} e_{\omega}^{2}-r_{2} K_{\Psi} e_{\Psi}^{2}-r_{4} K_{a} e_{a}^{2}-r_{4} K_{b} e_{b}^{2}-\frac{\varepsilon}{J} e_{\mathrm{T}}^{2}+\frac{\varepsilon}{r_{3}}\left(\frac{\varepsilon K_{\omega}}{J}+r_{1}\right) e_{\omega}^{2} \\
& +e_{a}\left[r_{4} u_{a}-r_{1} e_{\omega} k_{\mathrm{T}} \psi_{b}+2 r_{2} e_{\Psi} M R_{\mathrm{r}} \psi_{a}-\frac{\varepsilon r_{4} e_{\omega}}{r_{3}} \frac{L_{\mathrm{o}} \psi_{b}\left(K_{\omega}-D\right)}{\Psi^{2} k_{\mathrm{T}} J}+\frac{\varepsilon e_{\omega}}{r_{3} J} \varepsilon k_{\mathrm{T}} \psi_{b}\right] \\
& +e_{b}\left[r_{4} u_{b}+r_{1} e_{\omega} k_{\mathrm{T}} \psi_{a}+2 r_{2} e_{\Psi} M R_{\mathrm{r}} \psi_{b}+\frac{\varepsilon r_{4} e_{\omega}}{r_{3}} \frac{L_{\mathrm{o}} \psi_{a}\left(K_{\omega}-D\right)}{\Psi^{2} k_{\mathrm{T}} J}-\frac{\varepsilon r_{4} e_{\omega}}{r_{3} J} \varepsilon k_{\mathrm{T}} \psi_{a}\right]
\end{aligned}
$$

By examining the structure of (39) and completing the stability analysis, we now design the auxiliary control terms $u_{a}$ and $u_{b}$ inside the control input terms in (34) to cancel the sign-indefinite terms in (39). If $u_{a}$ and $u_{b}$ are chosen as

$$
\begin{aligned}
& u_{a}=\frac{1}{r_{4}}\left(r_{1} e_{\omega} k_{\mathrm{T}} \psi_{b}-2 r_{2} e_{\Psi} M R_{\mathrm{r}} \psi_{a}+r_{4} \frac{\varepsilon e_{\omega}}{r_{3}} \frac{L_{\mathrm{o}} \psi_{b}\left(K_{\omega}-D\right)}{\Psi^{2} k_{\mathrm{T}} J}\right)-\frac{\varepsilon e_{\omega}}{r_{3} J} \varepsilon k_{\mathrm{T}} \psi_{b} \\
& u_{b}=\frac{1}{r_{4}}\left(-r_{1} e_{\omega} k_{\mathrm{T}} \psi_{a}-2 r_{2} e_{\Psi} M R_{\mathrm{r}} \psi_{b}-r_{4} \frac{\varepsilon e_{\omega}}{r_{3}} \frac{L_{\mathrm{o}} \psi_{b}\left(K_{\omega}-D\right)}{\Psi^{2} k_{\mathrm{T}} J}\right)+\frac{\varepsilon e_{\omega}}{r_{3} J} \varepsilon k_{\mathrm{T}} \psi_{a}
\end{aligned}
$$

Then the control laws given by (34) and (40) together with the payload update law given by (38) will readily imply

$$
\dot{V}=-\left(r_{1} K_{\omega}-\frac{\varepsilon}{r_{3}} \frac{\varepsilon K_{\omega}}{J}+\frac{\varepsilon r_{1}}{r_{3}}\right) e_{\omega}^{2}-r_{2} K_{\Psi} e_{\Psi}^{2}-r_{4} K_{a} e_{a}^{2}-r_{4} K_{b} e_{b}^{2}-\frac{\varepsilon}{J} e_{\mathrm{T}}^{2}
$$

Let $\varepsilon$ be sufficiently small such that

$$
\left(r_{1} K_{\omega}-\frac{\varepsilon}{r_{3}} \frac{\varepsilon K_{\omega}}{J}+\frac{\varepsilon r_{1}}{r_{3}}\right)>0
$$

then there exists $K_{v}>0$ such that (41) implies the following:

$$
\dot{V} \leqslant-K_{v}\left\|\left[e_{w}, e_{\Psi}, e_{a}, e_{b}, e_{\mathrm{T}}\right]^{\mathrm{T}}\right\|^{2}
$$

Consequently, we know the signals $\left(e_{\omega}, e_{\Psi}, e_{a}, e_{b}, e_{\mathrm{T}}\right)$ in the closed-loop system approach to zero exponentially in time $t$ via Lyapunov exponential stability theory. 


\subsection{Stability of the overall system}

According to the above observer and control design, the following theorems will formally state all the detailed design procedure seen so far. Also, the consequences of the developed mechanism are clearly summarized and all the proofs can in fact be referenced from the previous sections.

\section{Theorem 1}

Consider an induction motor whose dynamics is governed by (1) under assumptions (A1)-(A5) If the stator current observers and the rotor flux observers are designed as

$$
\begin{aligned}
& L_{\mathrm{o}} \dot{\hat{I}}_{a}=k_{0} \tilde{I}_{a}-\left(M \hat{R}_{\mathrm{r}}+\beta_{1}\right) I_{a}+\hat{R}_{\mathrm{r}} \hat{\psi}_{a}+\beta_{2} \hat{\omega}_{\mathrm{r}} \hat{\psi}_{b}+\beta_{3} V_{a}+v_{1} \\
& L_{\mathrm{o}} \dot{\hat{I}}_{b}=k_{0} \tilde{I}_{b}-\left(M \hat{R}_{\mathrm{r}}+\beta_{1}\right) I_{b}-\beta_{2} \hat{\omega}_{\mathrm{r}} \hat{\psi}_{a}+\hat{R}_{\mathrm{r}} \hat{\psi}_{b}+\beta_{3} V_{b}+v_{2} \\
& L_{\mathrm{r}} \dot{\hat{\psi}}_{a}=-\hat{R}_{\mathrm{r}} \hat{\psi}_{a}-M \hat{R}_{\mathrm{r}} I_{a}-\beta_{2} \hat{\omega}_{\mathrm{r}} \hat{\psi}_{b}+v_{3} \\
& L_{\mathrm{r}} \dot{\hat{\psi}}_{b}=-\hat{R}_{\mathrm{r}} \hat{\psi}_{b}+M \hat{R}_{\mathrm{r}} I_{b}+\beta_{2} \hat{\omega}_{\mathrm{r}} \hat{\psi}_{a}+v_{4}
\end{aligned}
$$

where

$$
\begin{aligned}
& \hat{R}_{\mathrm{r}}=R_{\mathrm{r} n}+\hat{\theta}_{1} \\
& \hat{\omega}_{\mathrm{r}}=\omega_{\mathrm{rd}}+\hat{\theta}_{2}
\end{aligned}
$$

then the observed speed and fluxes of the rotor $\hat{\omega}_{\mathrm{r}}$ will be driven to approach to actual speed $\omega_{\mathrm{r}}$ and fluxes $\left(\psi_{a}, \psi_{b}\right)$, and the estimated rotor resistance will be also driven to approach to actual rotor resistance by the following primary law:

$$
\begin{aligned}
& v_{1}=-\frac{\hat{R}_{\mathrm{r}}}{L_{\mathrm{r}}}\left(L_{\mathrm{o}} \tilde{I}_{a}-\zeta_{a}\right)-\frac{\beta_{2} \hat{\omega}_{\mathrm{r}}}{L_{\mathrm{r}}}\left(L_{\mathrm{o}} \tilde{I}_{b}-\zeta_{b}\right) \\
& v_{2}=-\frac{\hat{R}_{\mathrm{r}}}{L_{\mathrm{r}}}\left(L_{\mathrm{o}} \tilde{I}_{b}-\zeta_{b}\right)+\frac{\beta_{2} \hat{\omega}_{\mathrm{r}}}{L_{\mathrm{r}}}\left(L_{\mathrm{o}} \tilde{I}_{a}-\zeta_{a}\right) \\
& v_{3}=-k_{0} \tilde{I}_{a}+\frac{L_{\mathrm{o}}}{L_{\mathrm{r}}}\left(\hat{R}_{\mathrm{r}} \tilde{I}_{a}+\beta_{2} \hat{\omega}_{\mathrm{r}} \tilde{I}_{b}\right) \\
& v_{4}=-k_{0} \tilde{I}_{b}+\frac{L_{\mathrm{o}}}{L_{\mathrm{r}}}\left(\hat{R}_{\mathrm{r}} \tilde{I}_{b}-\beta_{2} \hat{\omega}_{\mathrm{r}} \tilde{I}_{a}\right)
\end{aligned}
$$

And the auxiliary control law is as follows:

$$
\begin{aligned}
& \dot{\eta}_{a}=-\frac{1}{k_{\eta}}\left(\frac{\hat{R}_{\mathrm{r}}}{L_{\mathrm{r}} L_{\mathrm{o}}} \tilde{I}_{a}-\frac{\beta_{2} \hat{\omega}_{\mathrm{r}}}{L_{\mathrm{r}} L_{\mathrm{o}}} \tilde{I}_{b}\right) \\
& \dot{\eta}_{b}=-\frac{1}{k_{\eta}}\left(\frac{\hat{R}_{\mathrm{r}}}{L_{\mathrm{r}} L_{\mathrm{o}}} \tilde{I}_{b}-\frac{\beta_{2} \hat{\omega}_{\mathrm{r}}}{L_{\mathrm{r}} L_{\mathrm{o}}} \tilde{I}_{a}\right)
\end{aligned}
$$


with $k_{\eta}>0$, and the adaptation laws are designed as

$$
\begin{aligned}
& \dot{\hat{\theta}}_{1}=\left\{\begin{array}{cc}
\frac{1}{k_{R}}\left(\frac{\Omega_{11}}{L_{\mathrm{o}}} \tilde{I}_{a}+\frac{\Omega_{12}}{L_{\mathrm{o}}} \tilde{I}_{b}\right) & \text { if } \hat{\theta}_{1}(t)>-R_{\mathrm{r} n} \\
\varepsilon & \text { if } \hat{\theta}_{1}(t) \leqslant-R_{\mathrm{r} n}
\end{array}\right. \\
& \dot{\hat{\theta}}_{2}=\frac{1}{k_{\omega}}\left(\frac{\Omega_{21}}{L_{0}} \tilde{I}_{a}+\frac{\Omega_{22}}{L_{0}} \tilde{I}_{b}\right)
\end{aligned}
$$

for some constants

$$
k_{\omega}, k_{R}, \varepsilon>0
$$

If we use $\psi_{a}, \psi_{b}, \omega_{\mathrm{r}}$, and $R_{\mathrm{r}}$ estimated values provided by adaptive observer in Theorem 1, we obtain the adaptive feedback control theorem as follows:

\section{Theorem 2}

Consider an induction motor whose dynamics are governed by (1) under assumptions (A1)-(A5). If the rotor flux observers and the rotor resistance estimator are designed as in Theorem 1, then the mechanical angular speed of the rotor $\hat{\omega}_{r}$ and the rotor fluxes $\hat{\Psi}$ will be driven to approach to a bounded smooth speed command $\omega_{\text {rd }}$ and fluxes command $\Psi_{\mathrm{d}}$ with unknown load torque by the following primary control law:

$$
\begin{aligned}
& V_{a}=\frac{1}{\beta_{3}}\left(M \hat{R}_{\mathrm{r}} I_{a}+\beta_{1} I_{a}-\hat{R}_{\mathrm{r}} \hat{\psi}_{a}-\beta_{2} \hat{\omega}_{\mathrm{r}} \hat{\psi}_{b}+\Omega_{3}-K_{a} \hat{e}_{a}+u_{a}\right) \\
& V_{b}=\frac{1}{\beta_{3}}\left(M \hat{R}_{\mathrm{r}} I_{b}+\beta_{1} I_{b}-\hat{R}_{\mathrm{r}} \hat{\psi}_{b}+\beta_{2} \hat{\omega}_{\mathrm{r}} \hat{\psi}_{a}+\Omega_{4}-K_{b} \hat{e}_{b}+u_{b}\right)
\end{aligned}
$$

for some positive constants $K_{\omega}, K_{\Psi}, K_{a}$ and $K_{b}$, where $\Omega_{3}$ and $\Omega_{4}$ are defined as in (32), the auxiliary control inputs are designed as follows:

$$
\begin{aligned}
& u_{a}=\frac{1}{r_{4}}\left(r_{1} \hat{e}_{\omega} k_{\mathrm{T}} \hat{\psi}_{b}-2 r_{2} \hat{e}_{\Psi} M \hat{R}_{\mathrm{r}} \hat{\psi}_{a}+r_{4} \frac{\varepsilon \hat{e}_{\omega}}{r_{3}} \frac{L_{\mathrm{o}} \hat{\psi}_{b}\left(K_{\omega}-D\right)}{\hat{\Psi}^{2} k_{\mathrm{T}} J}\right)-\frac{\varepsilon \hat{e}_{\omega}}{r_{3} J} \varepsilon k_{\mathrm{T}} \hat{\psi}_{b} \\
& u_{b}=\frac{1}{r_{4}}\left(-r_{1} \hat{e}_{\omega} k_{\mathrm{T}} \hat{\psi}_{a}-2 r_{2} \hat{e}_{\Psi} M \hat{R}_{\mathrm{r}} \hat{\psi}_{b}-r_{4} \frac{\varepsilon \hat{e}_{\omega}}{r_{3}} \frac{L_{\mathrm{o}} \hat{\psi}_{a}\left(K_{\omega}-D\right)}{\hat{\Psi}^{2} k_{\mathrm{T}} J}\right)+\frac{\varepsilon \hat{e}_{\omega}}{r_{3} J} \varepsilon k_{\mathrm{T}} \hat{\psi}_{a}
\end{aligned}
$$


with some positive constants $r_{1}, r_{2}, r_{3}$ and $r_{4}$, and the payload adaptation law as

$$
\left.\hat{T}_{\mathrm{L}}=\frac{1}{r_{3}}\left(-r_{1} \hat{e}_{\omega}+r_{4} \hat{e}_{a} \frac{L_{\mathrm{o}} \hat{\psi}_{b}\left(K_{\omega}-D\right)}{\hat{\Psi}^{2} k_{\mathrm{T}} J}-r_{4} \hat{e}_{b} \frac{L_{\mathrm{o}} \hat{\psi}_{a}\left(K_{\omega}-D\right)}{\hat{\Psi}^{2} k_{\mathrm{T}} J}\right)+\frac{\varepsilon}{J}\left(k_{\mathrm{T}}\left(\hat{\psi}_{a} \hat{e}_{b}-\hat{\psi}_{b} \hat{e}_{a}\right)-K_{\omega} \hat{e}_{\omega}\right)\right)
$$

Note that $\hat{e}_{\omega}=\hat{\omega}_{\mathrm{r}}-\omega_{\mathrm{rd}}, \hat{e}_{\Psi}=\hat{\Psi}-\Psi_{\mathrm{d}}, \hat{e}_{a}=\hat{I}_{a}-I_{a \mathrm{~d}}, \hat{e}_{b}=\hat{I}_{b}-I_{b \mathrm{~d}}, \hat{\Psi}^{2}=\hat{\psi}_{a}^{2}+\hat{\psi}_{b}^{2}$, i.e. all the variables which are not measurable are all replaced by their observed values or estimates.

\section{Remark}

Referring to the error dynamics (30), (38) with proposed control (34), we can express the closed-loop system dynamics in a very concise manner as

$$
\dot{X}=F(X, y)
$$

where $X=\left[e_{\omega}, e_{\Psi}, e_{\mathrm{T}}, e_{a}, e_{b}\right]^{\mathrm{T}}$, and $y=\left[R_{\mathrm{r}}, \psi_{a}, \psi_{b}, \omega_{\mathrm{r}}\right]$, and $X$ converges to zero exponentially. However, since $y$ is not known a priori, the realistic controller shown in Theorem 2 adopts the estimate of $y$, namely, $\hat{y}$ so that the closed-loop system (42) becomes

$$
\dot{X}=F(X, \hat{y})
$$

and $\|F(X, \hat{y})-F(X, y)\| \leqslant k_{l}\|X\|\|\tilde{y}\|$, where $\tilde{y}=\hat{y}-y$ is always bounded from analysis of the observer. Therefore, by the following rearrangement

$$
\dot{X}=F(X, y)+(F(X, \hat{y})-F(X, y))
$$

and the fact that $X$ satisfies exponential convergence in (42), one can easily verify that $X$ remains to satisfy exponential stability provided $\|\tilde{y}\|$ is small enough.

Through the observer and controller design procedure, we have the actual currents approach to the desired ones, i.e. $I_{a} \rightarrow I_{a \mathrm{~d}}, I_{b} \rightarrow I_{b \mathrm{~d}}$. Because the desired flux $\left(\Psi_{\mathrm{d}}\right)$ and the desired speed $\left(\omega_{\mathrm{rd}}\right)$ are bounded trajectory, the desired currents are bounded. So, it can be verified through arguments of contradiction that the actual currents $\left(I_{a}, I_{b}\right)$ are indeed bounded at any time.

\section{SIMULATION AND EXPERIMENTAL RESULTS}

\subsection{Simulation results}

In this section, computer simulations of the controller designed above are done by SIMNON ${ }^{\mathrm{TM}} \mathrm{PCW}$ 2.0. The induction motor is set initially at rest with load torque $\left(T_{\mathrm{L}}=6+2 * \sin (0.5 t)\right)$ and is required to follow the various desired speed and flux trajectories with uncertainty in rotor resistance. The desired flux trajectory is described by the function $\left(\Psi_{\mathrm{d}}=0.5\left(1-\mathrm{e}^{-t}\right) \mathrm{Wb}\right)$, and the nominal value of rotor resistance is $0.53 \Omega$. We assume that the 
(a) Desired \& Actual Speed

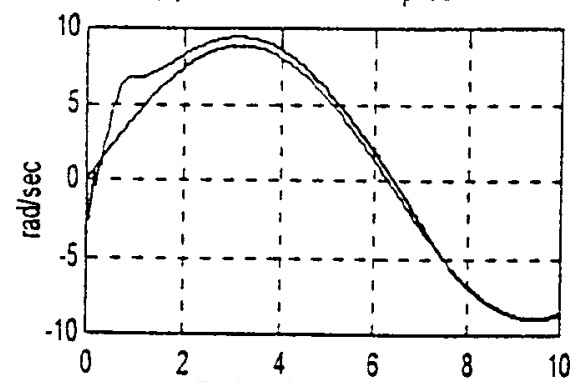

(c) Desired \& Actual Fluxes

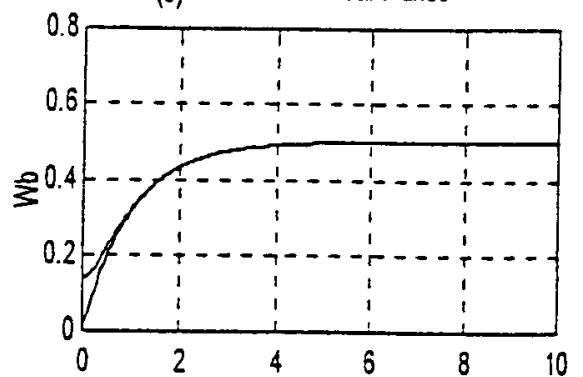

(e) ab Axis Currents

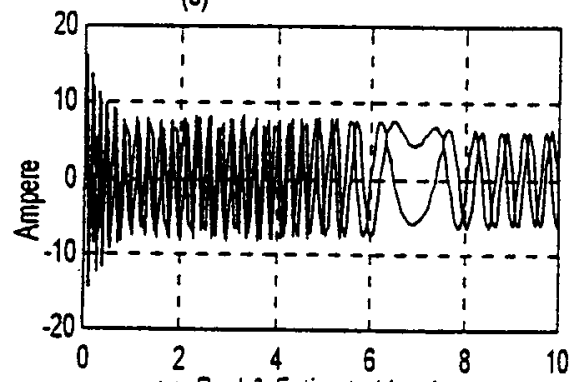

(g) Real 2 Estimated Load

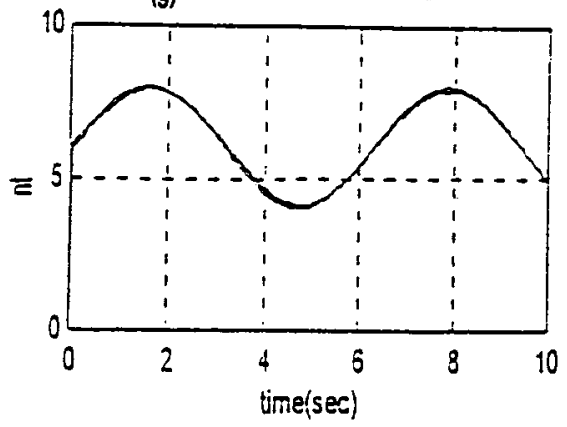

(b) Speed Tracking Error

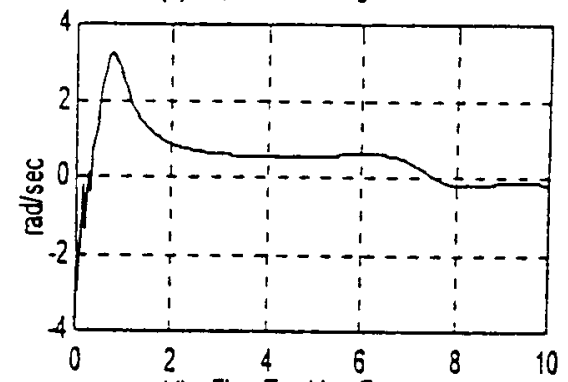

(d) Fiux Tracking Enor

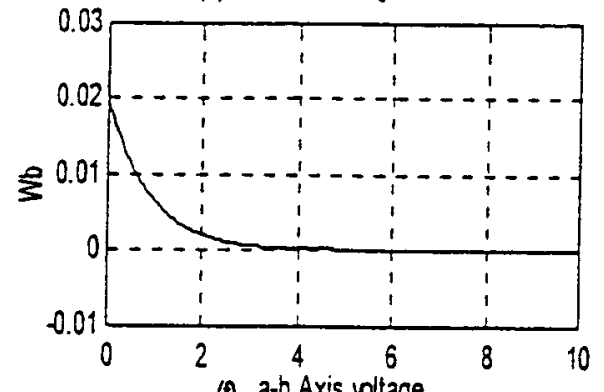

(i) $a-b$ Axis voltage

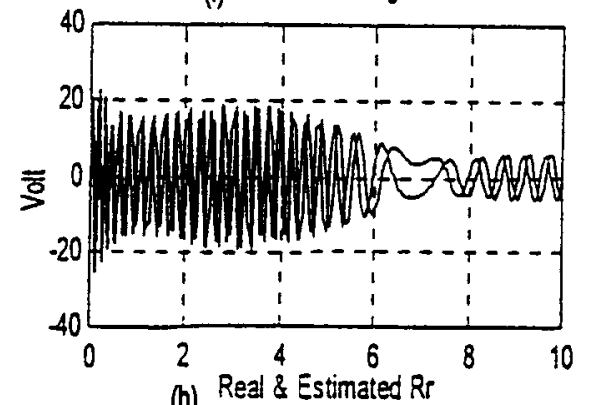

(h) Real \& Estimated Rr

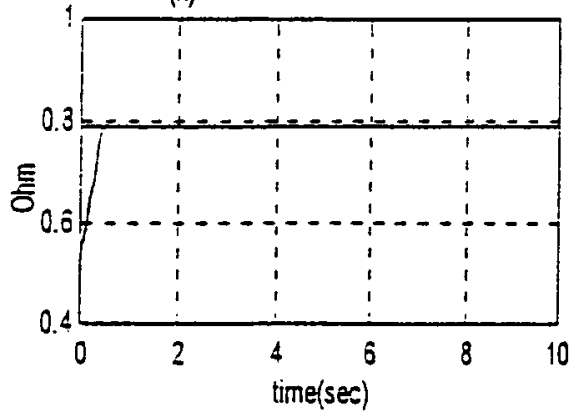

Figure 1. $\omega_{\text {rd }}=8.8496 \sin (0.5 t) \mathrm{rad}^{-1} / \mathrm{s}$ with $6+2 \sin (t) N t \cdot m$ load.

variation of the rotor resistance is up to 50 percent. The simulation results are shown in Figures 1 and 2. In each figure, part (a) shows the trajectories of the desired and the actual speed, whereas part (b) demonstrates the speed tracking error between the desired and actual speed. In a similar 
(a) Desired \& Actual Speed

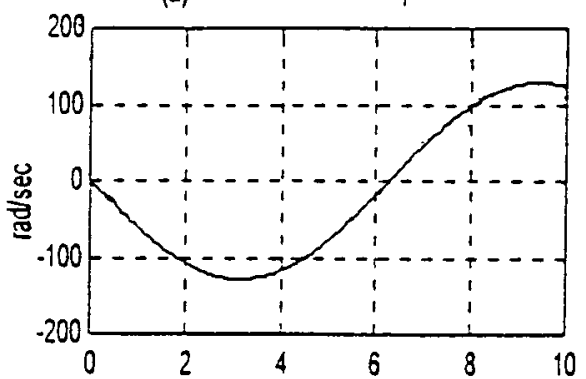

(c) Desired \& Actual Fluxes

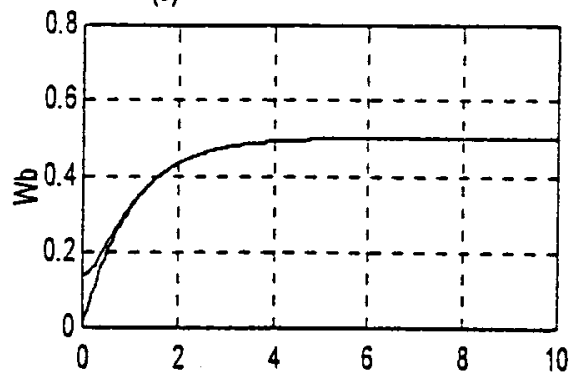

(e) $a-b$ Axis Currents

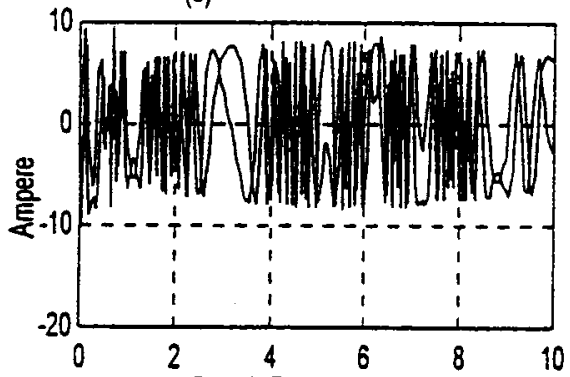

(g) Real \& Estimated Load

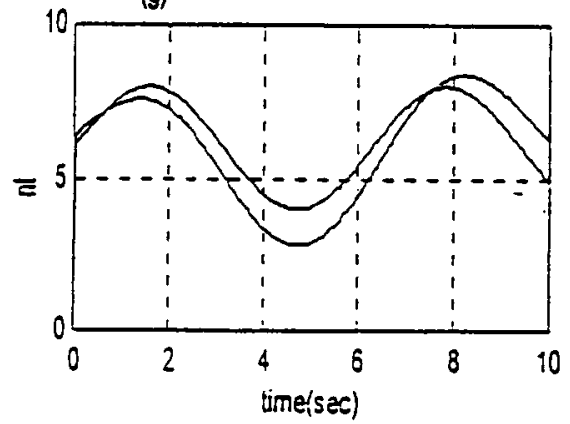

(b) Speed Tracking Error

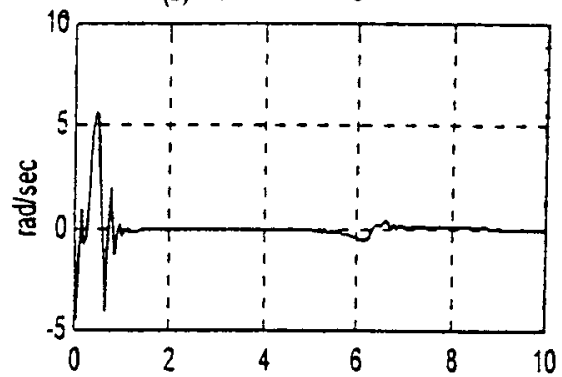

(d) Flux Tracking Enor

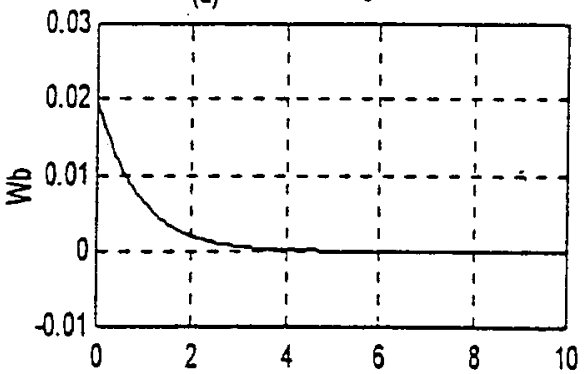

(f) ab Axis voltage

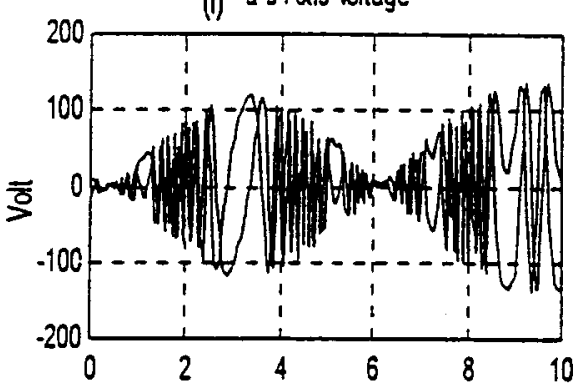

(h) Real \& Estimated Rr

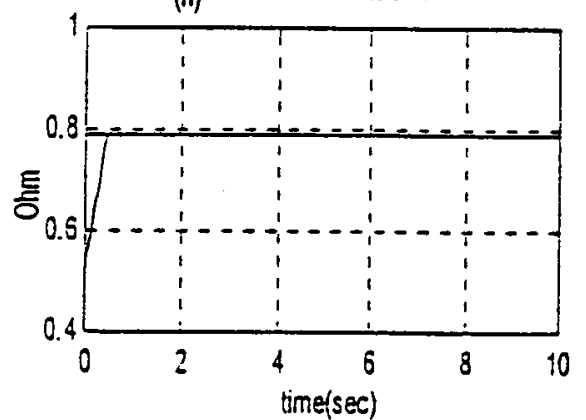

Figure 2. $\omega_{\text {rd }}=-128.496 \sin (0.5 t) \mathrm{rad}^{-1} / \mathrm{s}$ with $6+2 \sin (t) N t \cdot m$ load.

way, parts (c) and (d) show the trajectories of the desired and the actual flux and their error value, respectively. In addition, parts (e) and (f) respectively, depict the two phase stator currents and input voltages. Finally, part (g) and part (h) demonstrate the real and the estimated values of load 
(a) Desired and Actual Speed

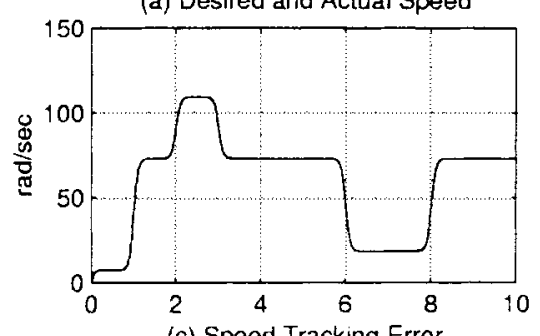

(c) Speed Tracking Error

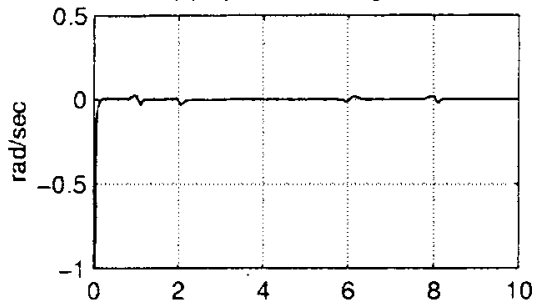

(e) Stator Current Magnitude

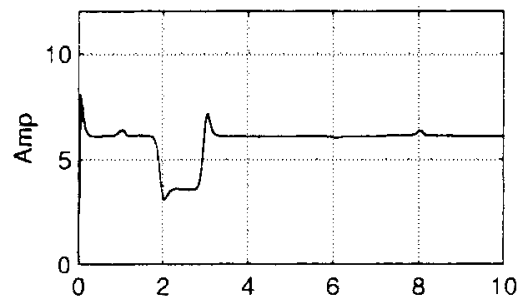

(g) Real \& Estimated Torque

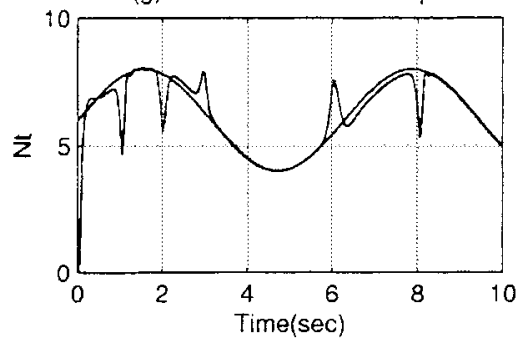

(b) Desire and Actual Flux Magnitude

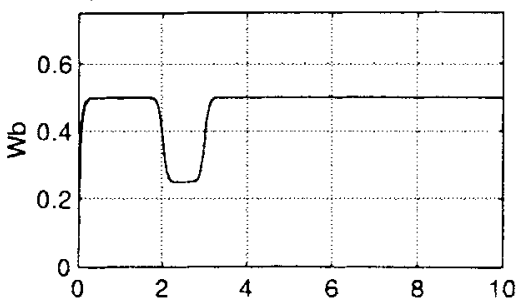

(d) Flux Magnitude Tracking Error
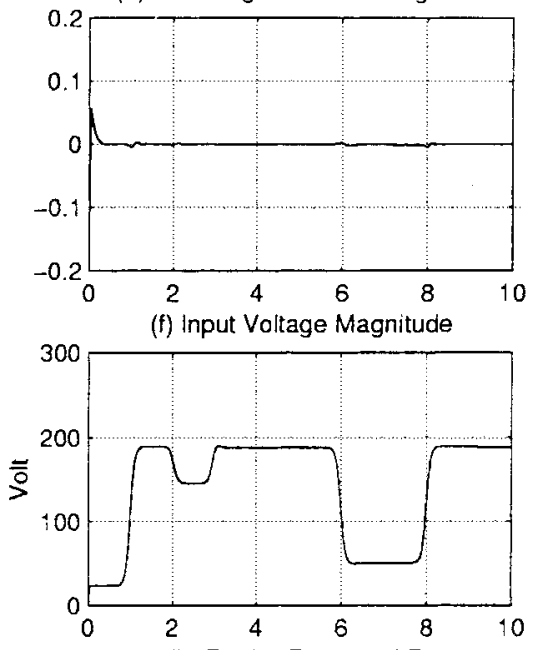

(h) Real \& Estimated $\mathrm{Rr}$

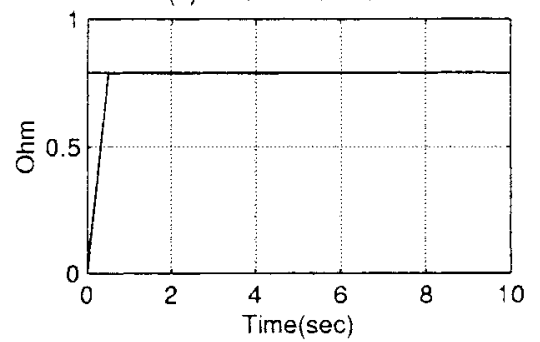

Figure 3. Benchmark test with $6+2 * \sin (t) N t \cdot m$ load.

torque and the rotor resistance, respectively. From the simulation results, we can see that the controller can control both the rotor speed and the rotor fluxes simultaneously without measuring the rotor flux and rotor speed signals.

In Figures 1 and 2, one can find that the tracking error and the estimation error do grow a little bit larger when the peak speed is higher. The reason is that the speed tracking error $\theta_{2}=\left(\omega_{\mathrm{r}}-\omega_{\mathrm{rd}}\right)$ is actually not changing slowly. 


\subsection{Simulations with benchmark specification}

We also perform computer simulations for the benchmark example. The rotor speed is required to change between those values: $\omega_{\text {nom }}, 0.1 \omega_{\text {nom }}, 0.25 \omega_{\text {nom }}, 1.5 \omega_{\text {nom }}$ during $t=[0,10] \mathrm{sec}$, where $\omega_{\text {nom }}=700 \mathrm{rev} \min ^{-1}$. The rotor flux is required to change from $\|\psi\|_{\text {nom }}$ to $0.5\|\psi\|_{\text {nom }}$ when the rotor speed is $1.5 \omega_{\text {nom }}$, where $\|\psi\|_{\text {nom }}=0.5 \mathrm{~Wb}$.

The modified benchmark assumption is shown below:

(B1) Measurable signals are the stator currents $\left(I_{a}, I_{b}\right)$.

(B2) Load torque $T_{\mathrm{L}}$ is constant, though unknown.

(B3) All parameters are known, except the rotor resistance $R_{\mathrm{r}}$.

(B4) Stator and rotor currents and stator voltages are constrained by $12 \mathrm{~A}$ and $300 \mathrm{~V}$.

(B5) The system begins with all zero initial conditions.

The speed tracking of the rotor speed and rotor flux for the benchmark test are shown in parts (c) and (d) of Figure 3. The parameter adaptation is shown in parts (g) and (h). The adaptation is achieved under the restriction of the stator currents and the stator input voltages from assumption (B4) of the benchmark test, which is also shown in parts (e) and (f) of Figure 3.

\subsection{Experimental results}

Experiments are done with a three horse power induction motor which is manufactured by TECO Co. Ltd. Taiwan. Parameters of the motor are listed in Table I.

The 3-HP induction motor used here is mounted with a 2000 pulse $^{-1}$ encoder. The $^{-1}$ controller is implemented by $\mathrm{C}$ language on a Pentium ${ }^{\mathrm{TM}}$ based PC. And the motor driver is implemented by MOSFET whose gate signals are generated by a $10 \mathrm{kHz}$ SPWM with $2 \cdot 2 \mu \mathrm{s}$ dead-time. The currents are measured by two NANA ${ }^{\mathrm{TM}}$ hall sensors. In order to check the performance, we have conducted three experiments with various kinds of sinusoidal speed commands as shown in Figure 4-6.

From the three figures, one can see that the speed tracking objective is achieved with different kinds of precision. In Figure 4, since the peak value is low and hence our previous assumption

Table I. Specifications and parameters of the motor.

\begin{tabular}{llll}
\hline \multicolumn{1}{c}{ Specification } & & Parameter \\
\hline Poles & 4 & $R_{\mathrm{s}}$ & $0.83 \Omega$ \\
Rated current & $8.6 \mathrm{~A}$ & $R_{\mathrm{r}}$ & $0.53 \Omega$ \\
Rated voltage & $220 \mathrm{~V}$ & $L_{\mathrm{s}}$ & $86.01 \mathrm{mH}$ \\
Rated frequency & $60 \mathrm{~Hz}$ & $L_{\mathrm{r}}$ & $86.01 \mathrm{mH}$ \\
Rated Speed & $1720 \mathrm{RPM}$ & $M$ & $82.59 \mathrm{mH}$ \\
Rated power & $2.2 \mathrm{~kW}$ & $J$ & $0.033 \mathrm{~kg} \mathrm{~m}^{2}$ \\
& & $D$ & $0.00825 \mathrm{~N} \mathrm{~m} \mathrm{~s}^{2}$ \\
\hline
\end{tabular}


(a) Desired \& Actual Speed
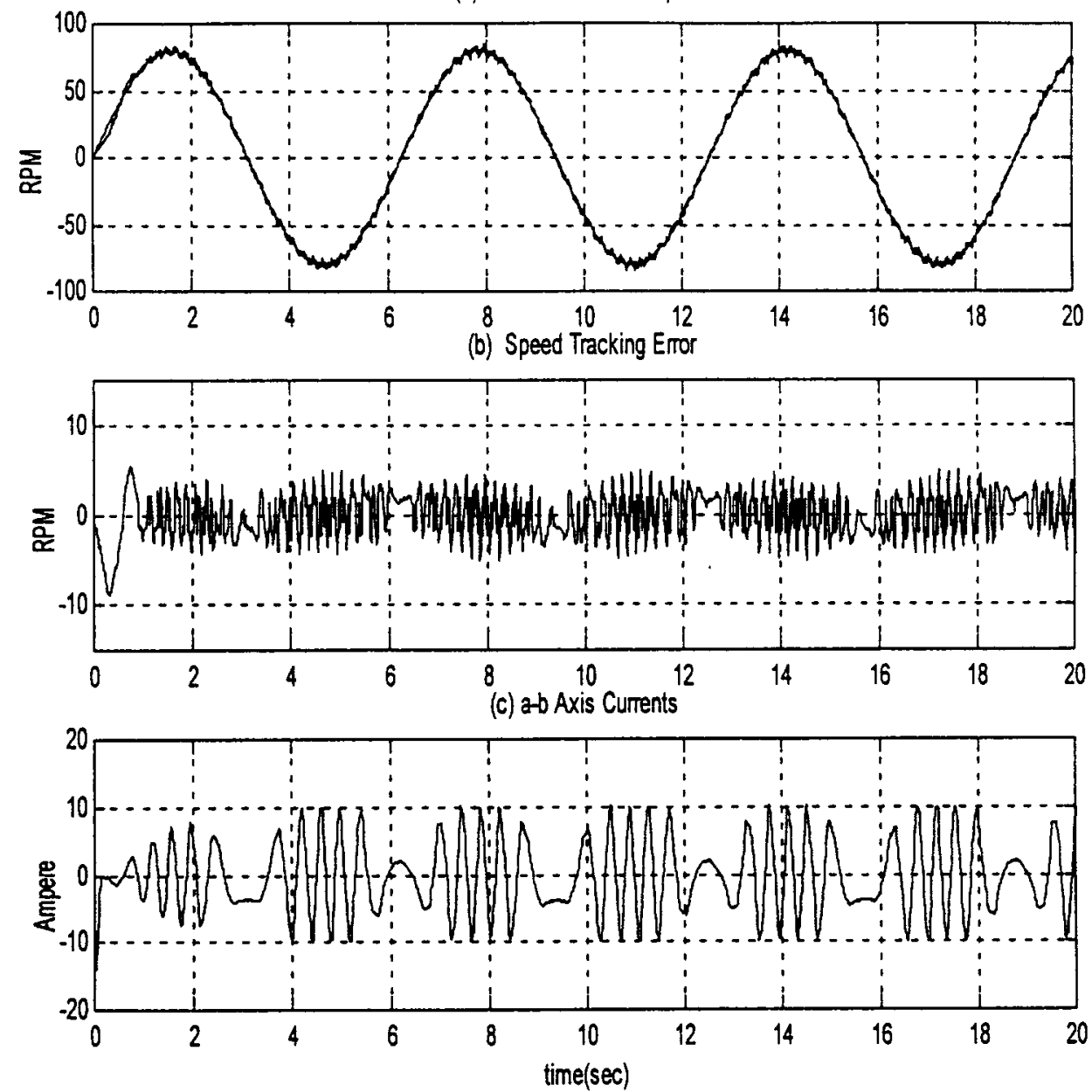

Figure 4. $\omega_{\text {rd }}=80 \sin (t)$ RPM with no load.

(A5) is more easily satisfied, the tracking error is quite moderate. But, in Figures 5 and 6, the tracking error is clearly larger but acceptable because the peak speed is higher. But it is noteworthy that Figure 6 demonstrates that the controller developed here indeed can serve as a speed servo, i.e. it is able to drive the motor to follow any kind of smooth speed command.

\section{CONCLUSION}

In this paper, we have presented a partial-state feedback adaptive sensorless speed and flux tracking controller for induction motors with fifth-order non-linear dynamic model which is 
(a) Desired \& Actual Speed

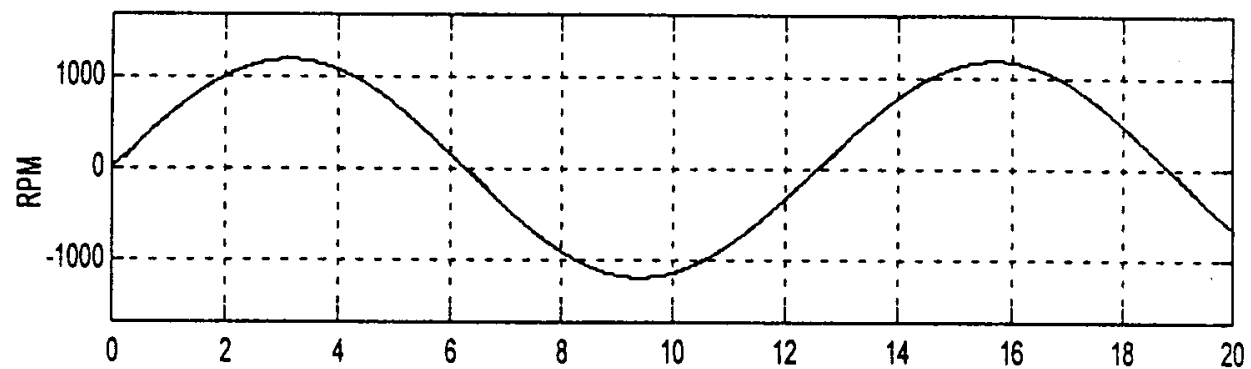

(b) Speed Tracking Error

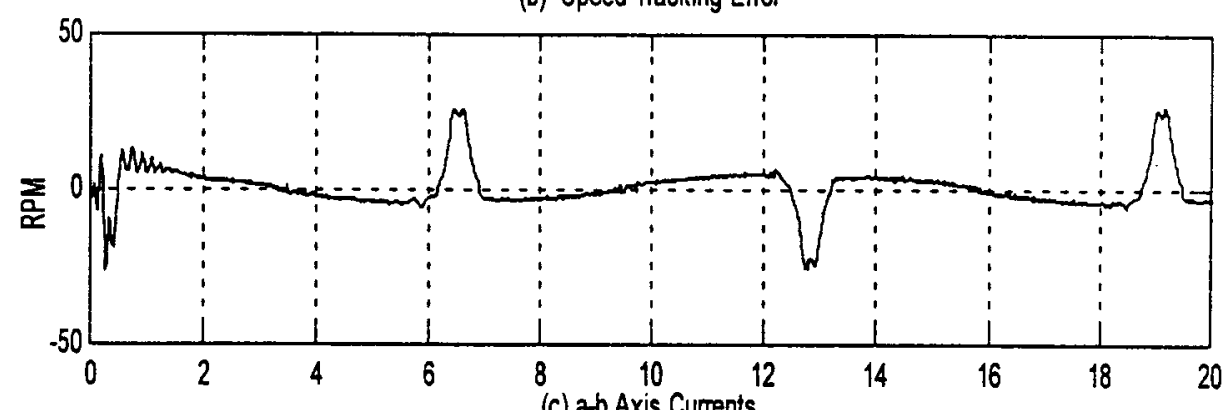

(c) $a b$ Axis Currents

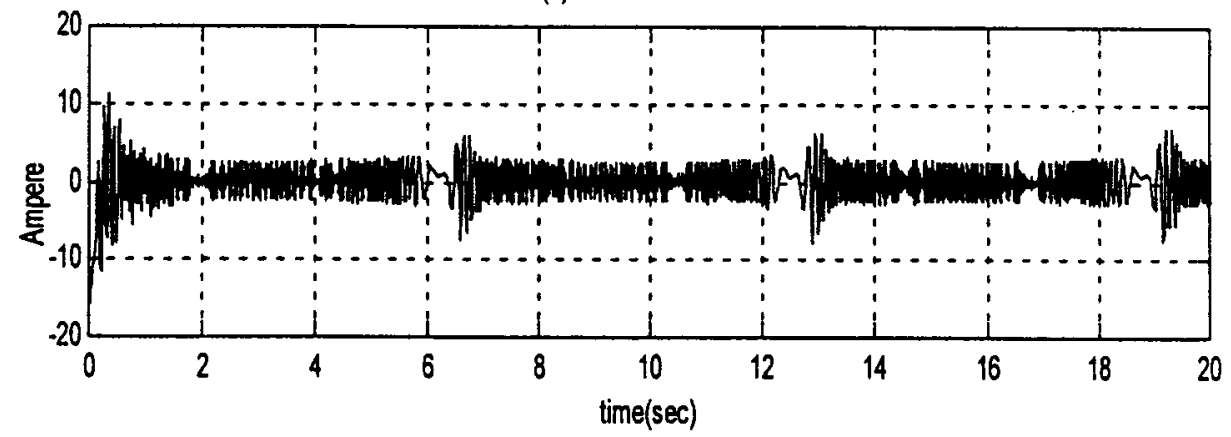

Figure 5. $\omega_{\mathrm{rd}}=1200 \sin (0.5 t) \mathrm{RPM}$ with no load.

actuated by a voltage source. We use an indirect adaptive control algorithm. First, we design an observer to get the actual values of fluxes, speed and rotor resistance. Then we design controller to achieve speed and flux tracking. The main contribution of the controller is that asymptotic tracking of rotor speed and rotor fluxes are achieved without the measurement of both the rotor fluxes and the rotor speed. Moreover, the variations of the rotor resistance and load torque are also taken into account. That is, only with the measurement of stator currents, this controller drives the induction motor to track given twice differentiable bounded speed and flux commands. 
(a) Desired \& Actual Speed
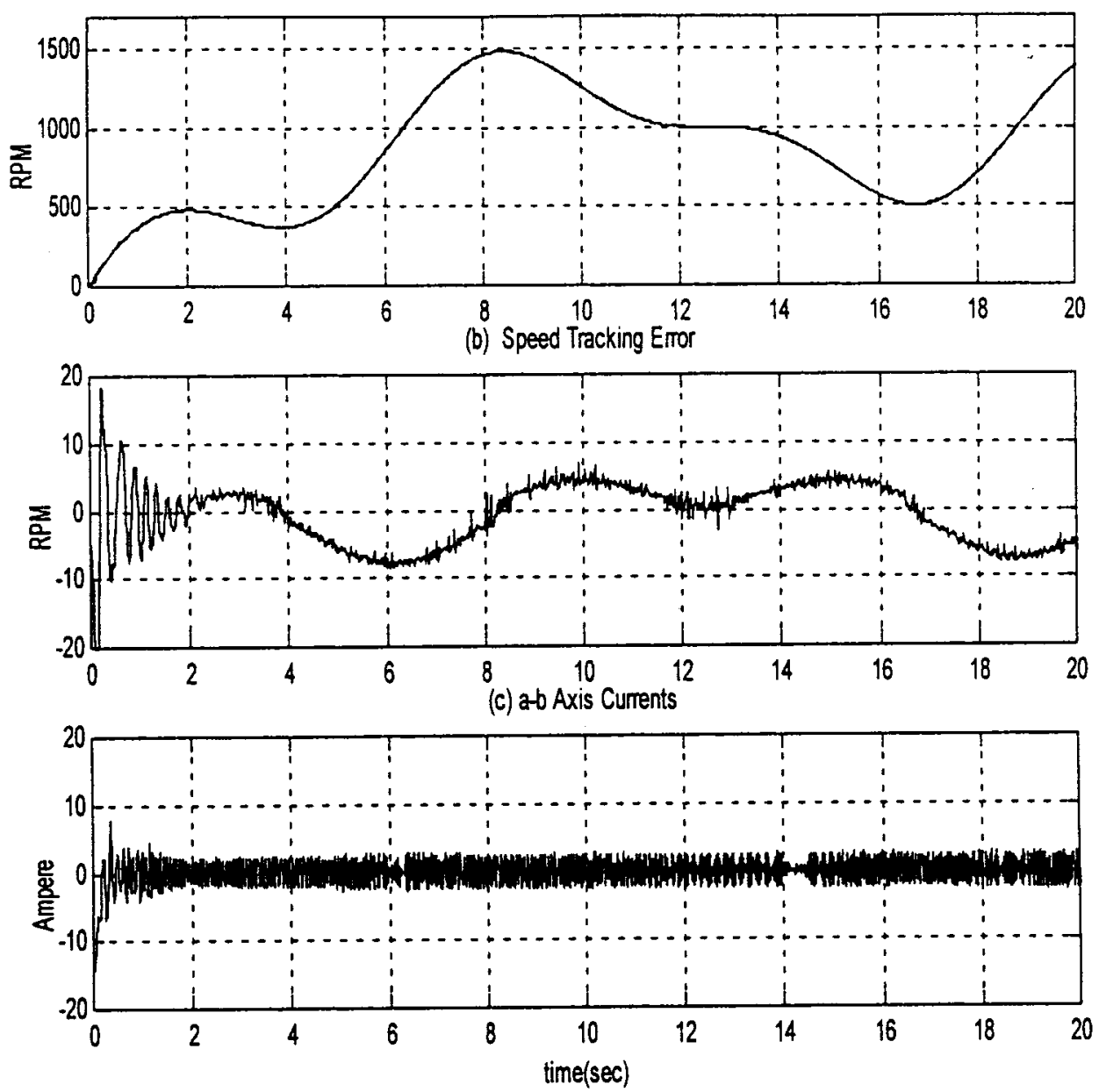

Figure 6. $\omega_{\text {rd }}=$ Hybrid function with no load.

\section{APPENDIX A}

Here, we derive an upper bound for the observation error of the rotor speed. First, from the mechanical part of the motor-load system

$$
\begin{gathered}
\dot{\omega}_{\mathrm{r}}+\frac{D}{J} \omega_{\mathrm{r}}=\frac{k_{\mathrm{T}}}{J}\left(\psi_{a} I_{b}-\psi_{b} I_{a}\right)-\frac{T_{\mathrm{L}}}{J} \\
\mathrm{e}^{(D / J)} \dot{\omega}_{\mathrm{r}}+\frac{D}{J} \mathrm{e}^{D / J} \omega_{\mathrm{r}}=\frac{1}{J}\left[k_{\mathrm{T}}\left(\psi_{a} I_{b}-\psi_{b} I_{a}\right)-T_{\mathrm{L}}\right] \mathrm{e}^{(D / J) t}
\end{gathered}
$$


then

$$
\begin{aligned}
\mathrm{e}^{D / J} \omega_{\mathrm{r}} & =\frac{1}{J} \int_{0}^{t}\left[k_{\mathrm{T}}\left(\psi_{a} I_{b}-\psi_{b} I_{a}\right)-T_{\mathrm{L}}\right] \mathrm{e}^{(D / J) \tau} \mathrm{d} \tau+\omega_{\mathrm{r}}(0) \\
& =\frac{T_{\mathrm{L}}}{J} \frac{J}{D}\left(1-\mathrm{e}^{(D / J) t}\right)+\frac{k_{\mathrm{T}}}{J} \int_{0}^{t}\left(\psi_{a} I_{b}-\psi_{b} I_{a}\right) \mathrm{e}^{(D / J) \tau} \mathrm{d} \tau
\end{aligned}
$$

or

$$
\omega_{\mathrm{r}}=\frac{T_{\mathrm{L}}}{D}\left(\mathrm{e}^{-(D / J) t}-1\right)+\frac{k_{\mathrm{T}}}{J} \int_{0}^{t}\left(\psi_{a} I_{b}-\psi_{b} I_{a}\right) \mathrm{e}^{-(D / J)(t-\tau)} \mathrm{d} \tau
$$

Since

$$
\frac{T_{\mathrm{L}}}{D}\left(\mathrm{e}^{-(D / J) t}-1\right) \leqslant 0, \quad \forall t \geqslant 0
$$

and the torque can be expressed in the vector form

$$
\left(\psi_{a} I_{b}-\psi_{b} I_{a}\right)=\mathbf{\Phi J I}
$$

where the matrices are defined as

$$
\begin{aligned}
& \mathbf{\Phi}=\left[\psi_{a}, \psi_{b}\right] \\
& \mathbf{J}=\left[\begin{array}{cc}
0 & 1 \\
-1 & 0
\end{array}\right] \\
& \mathbf{I}=\left[\begin{array}{c}
I_{a} \\
I_{b}
\end{array}\right]
\end{aligned}
$$

we can obtain the upper bound of the rotor speed as

$$
\begin{aligned}
\left|\omega_{\mathrm{r}}\right| & \leqslant \frac{k_{\mathrm{T}}}{J} \int_{0}^{t}\left|\left(\psi_{a} I_{b}-\psi_{b} I_{a}\right)\right| \mathrm{e}^{-(D / J)(t-\tau)} \mathrm{d} \tau \\
& \leqslant \frac{k_{\mathrm{T}}}{J} \int_{0}^{t} \mathbf{e}^{-(D / J)(t-\tau)} \mathrm{d} \tau \cdot\left(\left\|\boldsymbol{\Phi}_{\mathrm{t}}\right\|_{\infty} \cdot\left\|\mathbf{I}_{\mathbf{t}}\right\|_{\infty}\right) \\
& \leqslant \frac{k_{\mathrm{T}}}{D}\left(\left\|\boldsymbol{\Phi}_{\mathbf{t}}\right\|_{\infty} \cdot\left\|\mathbf{I}_{\mathbf{t}}\right\|_{\infty}\right)
\end{aligned}
$$

where the subscript $t$ denotes a truncated function which is defined as

$$
f_{k}(t)=\left\{\begin{array}{cc}
f(t) & 0 \leqslant t \leqslant k \\
0 & t>k
\end{array} \quad \forall k \geqslant 0\right.
$$


In the above, we see that the upper bound on the rotor speed is determined by the norm of rotor fluxes and the norm of stator currents. However, the rotor flux is unmeasurable, and therefore we try to find its upper bound associated with the currents. From the mathematical model of the motor in (1), we can write the dynamics of the rotor flux in the form

$$
\left[\begin{array}{c}
\dot{\psi}_{a} \\
\dot{\psi}_{b}
\end{array}\right]=\left[\begin{array}{cc}
-R_{\mathrm{r}} / L_{\mathrm{r}} & -\beta_{2} \omega_{\mathrm{r}} / L_{\mathrm{r}} \\
\beta_{2} \omega_{\mathrm{r}} / L_{\mathrm{r}} & -R_{\mathrm{r}} / L_{\mathrm{r}}
\end{array}\right]\left[\begin{array}{c}
\psi_{a} \\
\psi_{b}
\end{array}\right]+\left[\begin{array}{cc}
M R_{\mathrm{r}} / L_{\mathrm{r}} & 0 \\
0 & M R_{\mathrm{r}} / L_{\mathrm{r}}
\end{array}\right]\left[\begin{array}{c}
I_{a} \\
I_{b}
\end{array}\right]
$$

Consider

$$
V_{\psi}=\frac{1}{2}\left(\psi_{a}^{2}+\psi_{b}^{2}\right)
$$

then

$$
\begin{aligned}
\dot{V}_{\psi} & =\psi_{a} \dot{\psi}_{a}+\psi_{b} \dot{\psi}_{b} \\
& =\psi_{a}\left(-\frac{R_{\mathrm{r}}}{L_{\mathrm{r}}} \psi_{a}-\frac{\beta_{2} \omega_{\mathrm{r}}}{L_{\mathrm{r}}} \psi_{b}+\frac{M R_{\mathrm{r}}}{L_{\mathrm{r}}} I_{a}\right)+\psi_{b}\left(-\frac{R_{\mathrm{r}}}{L_{\mathrm{r}}} \psi_{b}+\frac{\beta_{2} \omega_{\mathrm{r}}}{L_{\mathrm{r}}} \psi_{a}+\frac{M R_{\mathrm{r}}}{L_{\mathrm{r}}} I_{b}\right) \\
& =-\frac{R_{\mathrm{r}}}{L_{\mathrm{r}}}\left(\psi_{a}^{2}+\psi_{b}^{2}\right)+\frac{M R_{\mathrm{r}}}{L_{\mathrm{r}}}\left(I_{a} \psi_{a}+I_{b} \psi_{b}\right) \\
& \leqslant-\frac{R_{\mathrm{r}}}{L_{\mathrm{r}}}\left(\psi_{a}^{2}+\psi_{b}^{2}\right)+\frac{R_{\mathrm{r}}}{2 L_{\mathrm{r}}}\left(\psi_{a}^{2}+\psi_{b}^{2}\right)+\frac{M^{2} R_{\mathrm{r}}}{2 L_{\mathrm{r}}}\left(I_{a}^{2}+I_{b}^{2}\right) \\
& =-\frac{R_{\mathrm{r}}}{2 L_{\mathrm{r}}}\left(\psi_{a}^{2}+\psi_{b}^{2}\right)+\frac{M^{2} R_{\mathrm{r}}}{2 L_{\mathrm{r}}}\left(I_{a}^{2}+I_{b}^{2}\right)
\end{aligned}
$$

so that

$$
\dot{V}_{\psi}+\frac{R_{\mathrm{r}}}{L_{\mathrm{r}}} V_{\psi} \leqslant \frac{M^{2} R_{\mathrm{r}}}{L_{\mathrm{r}}}\left(I_{a}^{2}+I_{b}^{2}\right)
$$

Following the procedure as that in the derivation for the upper bound on the rotor speed, the upper bound on $V_{\psi}$ is

$$
\begin{aligned}
V_{\psi} & \leqslant \frac{M^{2} R_{\mathrm{r}}}{2 L_{\mathrm{r}}} \int_{0}^{t} \mathrm{e}^{-\left(R_{\mathrm{r}} / L_{\mathrm{r}}\right)(t-\tau)}\left(I_{a}^{2}+I_{b}^{2}\right) \mathrm{d} \tau+\mathrm{e}^{-\left(R_{\mathrm{r}} / 2 L_{\mathrm{r}}\right)} V_{\psi}(0) \\
& \leqslant \frac{M^{2} R_{\mathrm{r}}}{2 L_{\mathrm{r}}} \int_{0}^{t} \mathrm{e}^{-\left(R_{\mathrm{r}} / 2 L_{\mathrm{r}}\right)(t-\tau)} \mathrm{d} \tau \cdot\left\|\mathbf{I}_{\mathrm{t}}\right\|_{\infty}^{2}+V_{\psi}(0) \\
& \leqslant \frac{M^{2}}{2}\left\|\mathbf{I}_{\mathrm{t}}\right\|_{\infty}^{2}+V_{\psi}(0) \quad \forall t \geqslant 0
\end{aligned}
$$


Since $V_{\psi}$ is the norm of the rotor flux, then we can find that

$$
\|\boldsymbol{\Phi}\|_{\infty}^{2} \leqslant M^{2}\left\|\mathbf{I}_{\mathbf{t}}\right\|_{\infty}^{2}+2 V_{\psi}(0)
$$

where

$$
V_{\psi}(0)=\frac{1}{2}\left(\psi_{a}^{2}(0)+\psi_{b}^{2}(0)\right) \leqslant \frac{1}{2 L_{\mathrm{r}}^{2}}\left(\delta_{a}^{2}+\delta_{b}^{2}\right)
$$

where

$$
\begin{aligned}
& \psi_{a}^{2}(0) \leqslant \delta_{a}^{2} \\
& \psi_{b}^{2}(0) \leqslant \delta_{b}^{2}
\end{aligned}
$$

Then from (A1) and (A2), we can associate the upper bound of the rotor speed with the norm of the stator currents as

$$
\begin{aligned}
\left|\omega_{\mathrm{r}}\right| & \leqslant \frac{k_{\mathrm{T}}}{D}\left\|\mathbf{I}_{\mathrm{t}}\right\|_{\infty} \sqrt{M^{2}\left\|\mathbf{I}_{\mathrm{t}}\right\|_{\infty}^{2}+2 V_{\psi}(0)} \\
& \leqslant \frac{k_{\mathrm{T}} M}{D}\left\|\mathbf{I}_{\mathrm{t}}\right\|_{\infty}^{2}+\frac{k_{\mathrm{T}}}{D} \frac{1}{L_{\mathrm{r}}} \sqrt{\delta_{a}^{2}+\delta_{b}^{2}}\left\|\mathbf{I}_{\mathrm{t}}\right\|_{\infty}
\end{aligned}
$$

Since the upper bound on the rotor speed is found, the upper bound on the observation error of the rotor speed can be found as

$$
\begin{aligned}
\left|\omega_{\mathrm{r}}-\hat{\omega}_{\mathrm{r}}\right| & \leqslant\left|\omega_{\mathrm{r}}\right|+\left|\hat{\omega}_{\mathrm{r}}\right| \\
& \leqslant \frac{k_{\mathrm{T}} M}{D}\left\|\mathbf{I}_{\mathrm{t}}\right\|_{\infty}^{2}+\frac{k_{\mathrm{T}}}{2(D)} \frac{1}{L_{\mathrm{r}}} \sqrt{\delta_{a}^{2}+\delta_{b}^{2}}\left\|\mathbf{I}_{\mathrm{t}}\right\|_{\infty}+\left|\hat{\omega}_{\mathrm{r}}\right|
\end{aligned}
$$

\section{APPENDIX B}

Theorem Al [17]

The second differential equation has the form

$$
\begin{gathered}
\dot{x}_{1}(t)=A(t) x_{1}(t)+u^{\mathrm{T}}(t) x_{2}(t) \\
\dot{x}_{2}(t)=-u(t) x_{1}(t)
\end{gathered}
$$

where $x_{1}:[0, \infty) \rightarrow \mathbf{R}^{m}, x_{2}:[0, \infty) \rightarrow \mathbf{R}^{n}, A(t)$ and $u(t)$ are, respectively, $n \times n$ and $n \times m$ matrices of bounded piecewise-continuous functions, and $A(t)+A^{\mathrm{T}}(t)$ is uniformly negative definite, that is, $A(t)+A^{\mathrm{T}}(t) \leqslant-Q<0$. Let $x=\left[x_{1}^{\mathrm{T}}, x_{2}^{\mathrm{T}}\right]^{\mathrm{T}}$. The equibrium point $x=0$ in Equation (B) is 
uniformly asymptotically stable if, and only if, positive constants $T_{0}, \delta_{0}$ and $\varepsilon_{0}$ exist with a $t_{2} \in[t$, $\left.t+T_{0}\right]$ such that for any unit vector $w \in \mathbf{R}^{m}$,

$$
\left\|\frac{1}{T_{0}} \int_{t_{2}}^{t_{2}+\delta_{0}} u^{\mathrm{T}}(\tau) w \mathrm{~d} \tau\right\| \geqslant \varepsilon_{0}, \quad \forall t \geqslant t_{0}
$$

When $\|\dot{u}(t)\|$ is uniformly bounded, the condition in Equation (B2) in Theorem 1 can be relaxed as given in Corollary 1.

Corollary $A 2$

If $u(t)$ is smooth, $\dot{u}(t)$ is uniformly bounded, and $u(t)$ satisfies the condition

$$
\frac{1}{T_{0}} \int_{t}^{t+T_{0}}\left\|u^{\mathrm{T}}(\tau) w\right\| \mathrm{d} \tau \geqslant \varepsilon_{0}, \quad \forall t \geqslant t_{0}
$$

for $t_{0} \in \mathbf{R}^{+}$, and positive constants $T_{0}$ and $\varepsilon_{0}$, and all unit vectors $\omega \in \mathbf{R}^{n}$, then the solution $x=0$ of Equation (B1) is u.a.s.

Proof: Let $\left\|x_{1}(t)\right\|<c\|x(t)\| \forall t \in\left[t_{0}, t_{0}+T_{0}\right]$, where $c \in(0,1)$. Since $u$ satisfies the condition in (B2), there exists a $t_{2} \in\left[t_{0}, t_{0}+T_{0}\right]$ so that

$$
\int_{t_{2}}^{t_{2}+\delta_{0}}\left\|u^{\mathrm{T}}(\tau) x_{2}(\tau)\right\| \mathrm{d} \tau \geqslant \varepsilon_{0} T_{0}\left\|x_{2}\left(t_{2}\right)\right\|-u_{\max }^{2} \delta_{0}^{2} \sup _{\tau \in\left[t_{2}, t_{2}+\delta_{0}\right]}\left\|x_{1}(\tau)\right\|
$$

Integrating the first equation over the interval $\left[t_{2}, t_{2}+\delta_{0}\right]$ in (B1) it follows that

$$
\left\|x_{1}\left(t_{2}+\delta_{0}\right)\right\| \geqslant\left[\varepsilon_{0} T_{0} \sqrt{1-c^{2}}-c\left(u_{\max }^{2} \delta_{0}^{2}+A_{\max } \delta_{0}+1\right)\right]\left\|x_{1}\left(t_{2}+\delta_{0}\right)\right\|
$$

since $\left\|x_{2}\left(t_{2}\right)\right\| \geqslant \sqrt{1-c^{2}}\left\|x_{2}\left(t_{2}\right)\right\|$ and $\left\|x_{2}\left(t_{2}\right)\right\| \geqslant\left\|x_{2}\left(t_{2}+\delta_{0}\right)\right\|$. If

$$
\begin{aligned}
c^{2} & =\frac{T_{0}^{2} \varepsilon_{0}^{2}}{T_{0}^{2} \varepsilon_{0}^{2}+(1+b)^{2}} \\
b & =u_{\max }^{2} \delta_{0}^{2}+A_{\max } \delta_{0}+1
\end{aligned}
$$

we have from Equation (B3)

$$
\left\|x_{1}\left(t_{2}+\delta_{0}\right)\right\| \geqslant c\left\|x\left(t_{2}+\delta_{0}\right)\right\|
$$

which is a contradiction of the assumption regarding $\left\|x_{1}(t)\right\|$. 
Integrating $\dot{V}(x)$ over an interval $\left[t_{1}, t_{1}+T\right] \subset\left[t_{0}, t_{0}+T_{0}\right]$, we obtain that

$$
\begin{aligned}
V\left(t_{1}\right)-V\left(t_{1}+T\right) & \geqslant \lambda_{Q-\min } \int_{t_{1}}^{t_{1}+T}\left\|x_{1}(\tau)\right\|^{2} \mathrm{~d} \tau \\
& \geqslant \frac{\lambda_{Q-\min }}{T}\left(\int_{t_{1}}^{t_{1}+T}\left\|x_{1}(\tau)\right\| \mathrm{d} \tau\right)^{2}
\end{aligned}
$$

where $\lambda_{Q-\min }$ is the minimum eigenvalue of $Q$. Further, by integrating the first equation in Equation (B1) over $\left[t_{1}, t_{1}+T\right]$

$$
\int_{t_{1}}^{t_{1}+T}\left\|x_{1}(\tau)\right\| \mathrm{d} \tau \geqslant T\left\|x_{1}\left(t_{1}\right)\right\|-T^{2} \mathrm{~d} \sqrt{V\left(t_{1}\right)}
$$

where $d=A_{\max }+u_{\max },\|A(t)\| \leqslant A_{\max }$ and $\|u(t)\| \leqslant u_{\max }$.

Choosing $t_{1}=t_{2}+\delta_{0}$, from Equation (B4), it follows that

$$
V\left(t_{1}+T\right) \leqslant(1-\gamma) V\left(t_{1}\right)
$$

where

$$
\gamma=\lambda_{Q-\min } T(c-T d)^{2}
$$

Let $T=\min \left(t_{0}+T_{1}, c / d\right)$, we have

$$
V\left(t_{0}+T_{0}\right) \leqslant V\left(t_{1}+T\right) \leqslant(1-\gamma) V\left(t_{1}\right) \leqslant(1-\gamma) V\left(t_{0}\right)
$$

Since $\lambda_{Q-\min } \leqslant 2, c \in(0,1)$ and $T d(c-T d)^{2} \leqslant 4 c^{3} / 27$, it follows that $\gamma \in\left(0,8 c^{3} / 27\right)$ and hence $x=0$ in Equation (B1) is u.a.s.

\section{NOMENCLATURE}

$\left\{V_{a}, V_{b}\right\} \quad$ stator voltages in the stationary reference frame

$\left\{I_{a}, I_{b}\right\} \quad$ stator currents in the stationary reference frame

$\left\{\psi_{a}, \psi_{b}\right\} \quad$ rotor fluxes in the stationary reference frame

$\omega_{\mathrm{r}} \quad$ mechanical angular speed of the rotor

$R_{\mathrm{s}} \quad$ stator resistance

$R_{\mathrm{r}} \quad$ rotor resistance

$L_{\mathrm{s}} \quad$ stator self-inductance

$L_{r} \quad$ Rotor self-inductance

$M \quad$ mutual inductance

$p \quad$ number of pole-pairs

$J \quad$ rotor inertia

D damping coefficient 


$\begin{array}{ll}T_{\mathrm{L}} & \text { load torque } \\ k_{\mathrm{T}} & \text { torque constant }\left(=\frac{3 p M}{2 L_{\mathrm{r}}}\right) \\ L_{\mathrm{o}} & \frac{L_{\mathrm{r}}^{2}}{M}\left(L_{\mathrm{s}}-\frac{M^{2}}{L_{\mathrm{r}}}\right) \\ \beta_{1} & \frac{R_{\mathrm{s}} L_{\mathrm{r}}^{2}}{M} \\ \beta_{2} & p L_{\mathrm{r}} \\ \beta_{3} & \frac{L_{\mathrm{r}}^{2}}{M}\end{array}$

\section{REFERENCES}

1. Leonhard W. Microcomputer control of high dynamic performance ac-drives-a survey, Automatica 1986; 22:1-19.

2. Luca AD, Ulivi G. Design of an exact nonlinear controller for induction motors. IEEE Transactions on Automatic Control 1989; 34(12):1304-1307.

3. Marino R, Peresada S, Valigi P. Adaptive input-output linearizing control of induction motors. IEEE Transactions on Automatic Control 1993; 38:208-221.

4. Bellini A, Figalli G, Ulivi G. Analysis and design of a microcomputer-based observer for an induction machine. Automatica 1988; 24:549-555.

5. Verghese GC, Sanders SR. Observers for flux estimation in induction machines. IEEE Transactions on Industrial Electronics 1988; 35:85-94.

6. Stephan J, Bodson M, Chiasson J. Real-time estimation of the parameters and fluxes of induction motors. IEEE Transactions on Industrial Applications 1994; 30(3):746-758.

7. Marino R, Peresada S, Tomei P. Exponentially convergent rotor resistance estimation for induction motors. IEEE Transactions on Industrial Electronics 1995; 42(5):508-515.

8. Hu J, Dawson DM. Adaptive control of induction motor systems despite rotor resistance uncertainty. Proceedings of the American Control Conference, June 1996, pp. 1397-1402.

9. Jung-Hua Yang, Wen-Hai Yu, Li-Chen Fu. Nonlinear observer-based adaptive tracking control for induction motors with unknown load. IEEE Transactions on Industrial Electronics 1995; 42(6):579-586.

10. Marino R, Peresada S, Tomei P. Adaptive observer-based control of induction motors with unknown rotor resistance. IEEE International Journal of Adaptive Control and Signal Processing 1996; 10:345-363.

11. Marino R, Peresada S, Tomei P. Global adaptive output feedback control of induction motors with uncertain rotor resistance. Proceedings of the 35th Conference on Decision and Control, 1996, pp. 4701-4706.

12. Ohtani T, Takada N, Tanaka K. Vector control of induction motor without shaft encoder. IEEE Transactions of Industrial Application 1992; 28(1):157-164.

13. Kubota H, Matsuse K. Speed sensorless field-oriented control of induction motor with rotor resistance adaptation. IEEE Transactions on Industrial Application 1994; 30(5):1219-1224.

14. Has C, Bettini A, Feraris L, Griva G, Profumo F. Comparison of different schemes without shaft sensors for field oriented control drives. Proceedings of the IEEE IECON'94, 1994, pp. 1579-1588.

15. Chin TH. Approaches for vector control of induction motor without speed sensors. Proceedings of IEEE IECON'94, 1994, pp. 1616-1620.

16. Krause PC. Analysis of Electric Machinery, McGraw-Hill: New York, 1987.

17. Morgan AP, Narendra KS. On the stability of nonautonomous differential equations $\dot{x}=[A+B(t)] x$ with skewsymmetric matrix B(t). SIAM Journal of Control and Optimization 1997; 15:163-176.

18. Espinosa G, Ortega R. State observers are unnecessary for induction motor control. Systems and Control Letters 1994; 23(5):315-323

19. Lee AM, Fu LC. Nonlinear adaptive speed and torque control of induction motors with unknown rotor resistance. Master Thesis, National Taiwan University Taiwan R.O.C., 1996.

20. Vidyasagar M. Nonlinear System Analysis. Prentice-Hall: Englewood Cliffs, NJ, 1992.

21. Narendra KS, Annaswamy AM. State Adaptive Systems. Prentice-Hall: Englewood Cliffs, NJ, 1989.

22. Hu J, Dawson DM, Qian Y. Position tracking control of an induction motor via parial state feedback. Automatica 1995; 31(7):989-1000. 